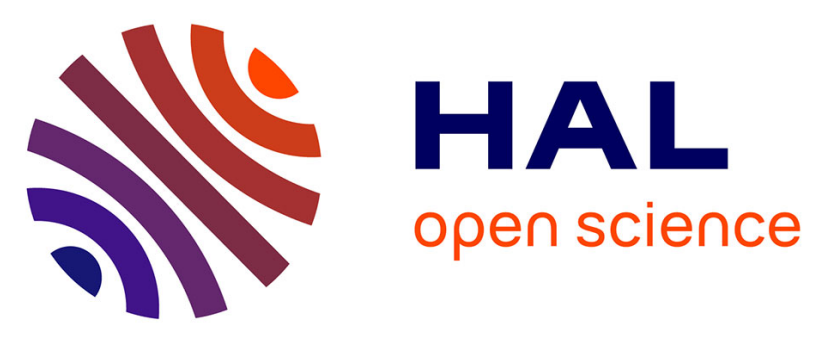

\title{
Archéométrie des déchets de production sidérurgique: moyens et méthodes d'identification des différents éléments de la chaîne opératoire directe
}

\author{
Philippe Fluzin, Alain Ploquin, Vincent Serneels
}

\section{- To cite this version:}

Philippe Fluzin, Alain Ploquin, Vincent Serneels. Archéométrie des déchets de production sidérurgique: moyens et méthodes d'identification des différents éléments de la chaîne opératoire directe. Gallia - Archéologie de la France antique, 2000, Mines et métallurgies en Gaule, 57, pp.101-121. 10.3406/galia.2000.3212 . hal-01902645

\section{HAL Id: hal-01902645 \\ https://hal.science/hal-01902645}

Submitted on 20 Jan 2020

HAL is a multi-disciplinary open access archive for the deposit and dissemination of scientific research documents, whether they are published or not. The documents may come from teaching and research institutions in France or abroad, or from public or private research centers.
L'archive ouverte pluridisciplinaire HAL, est destinée au dépôt et à la diffusion de documents scientifiques de niveau recherche, publiés ou non, émanant des établissements d'enseignement et de recherche français ou étrangers, des laboratoires publics ou privés.

\section{(ㅇ)(1) $\$$}

Distributed under a Creative Commons Attribution - NonCommercial - NoDerivatives| 4.0 


\title{
ARCHÉOMÉTRIE DES DÉCHETS DE PRODUCTION SIDÉRURGIQUE
}

\author{
Moyens et méthodes d'identification \\ des différents éléments de la chaîne opératoire directe
}

\author{
Philippe Fluzin, Alain Ploquin et Vincent SERneElS
}

\begin{abstract}
Mots-clés. Paleométallurgie du fer, bas fourneau, haut fourneau, scorie, laitier, grillage, réduction, épuration, forge, affinage, minerais, métallographie, pétrologie, typologie.
\end{abstract}

Key-words. Archaeometallurgy of iron, shaft furnace, blast furnace, slag, roasting, smelting, cleansing, smithing, finery, ores, metallography, petrology, typology.

Résumé. Cet article n'est pas un manuel d'archéométrie, mais un exposé des méthodes d'étude des produits et déchets de la sidérurgie ancienne, auxquels peuvent être confrontés les archéologues.

Les caractères des principales chaînes opératoires sidérurgiques anciennes et de leurs produits sont présentés après avoir rappelé quelques notions de base de minéralogie, de métallurgie et sur les transformations physico-chimiques.

Les principales méthodes d'étude utilisées en archéométrie appliquées aux productions et aux déchets, essentiellement celle de la chaîne du bas fourneau ou filière directe, sont discutées.

\begin{abstract}
This paper is not a handbook of archaeometry, but a statement of methods for the studies of ancient ironmaking wastes and products, for the archaeologists.

The features of the main iron making processes and their products are explained after some basic concepts of mineralogy, metallurgy and on physico-chemical processing.

The main methods of studies used in archaeometry applied to the productions and scraps are being discussed, especially the one about the low shaft furnace or direct process.
\end{abstract}

\section{NOTIONS PRÉLIMINAIRES}

Obtenir un objet en fer à partir de minerai exige une succession de transformations chimiques, physiques et mécaniques (processus physico-chimiques) mises en œuvre par divers procédés techniques. Cette succession d'opérations, le plus souvent dans différents ateliers, de la mine à l'objet, constitue la chaîne opératoire. Deux grands types de chaîne opératoire ont existé en Europe: la filière directe, celle du bas fourneau, et la filière indirecte, celle du haut fourneau.

En association avec les données archéologiques et historiques, les études archéométriques visent à reconstituer la chaîne opératoire (fig. 38). Nous ne considérerons pas ici les méthodes plus directement liées à la fouille d'ateliers (géophysique, étude des installations, tracéologie des impacts thermiques, répartitions des déchets, datations, anthracologie, etc.), ni à l'étude de 


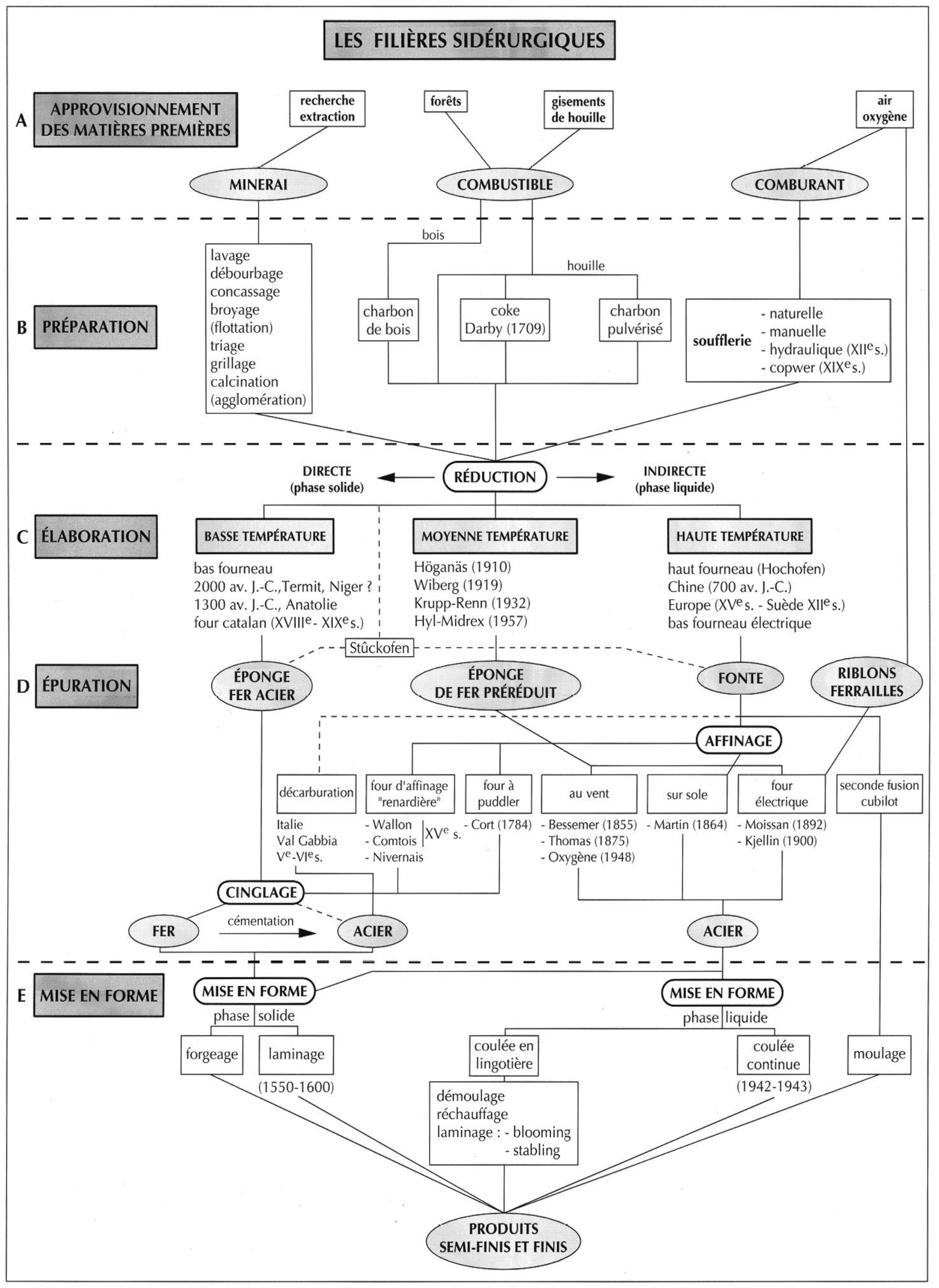

Fig. 38 - Les filières sidérurgiques et leur évolution historique (d'après P. Fluzin). 
l'extraction minière. Nous limiterons ce chapitre à l'étude en laboratoire des matériaux produits par l'extraction métallurgique, ou élaboration du métal à partir du minerai déjà préparé par le mineur, et par la mise en forme du métal ou forge.

La première démarche sera faite avec l'archéologue et comprendra un examen visuel de l'ensemble des matériaux: définition des types morphologiques et de leurs associations, relations avec les faits archéologiques, estimations plus ou moins quantitatives des types; puis sélection, en commun, des spécimens à étudier et plan de travail, en fonction des questions qui se posent.

Dans les faits, deux modes d'approches archéométriques différentes ont été élaborées séparément au sein de ce que l'on pourrait appeler « l'école française " qui tendent maintenant à se rapprocher, volontairement, avec toutes les difficultés inhérentes à des cultures scientifiques différentes. La première, historiquement, est l'approche par l'analyse métallographique, développée essentiellement par des métallurgistes. Initialement orientée quasi exclusivement vers l'étude des objets manufacturés et des rares lingots disponibles, elle s'est peu à peu intéressée au métal des déchets et des scories, puis aux scories. La seconde, de type pétrologique, plus récente malgré quelques précurseurs, est développée par quelques géologues, pétrographes et géochimistes qui se sont surtout intéressés aux matériaux de types «rocheux »: minerais, scories, parois (les métaux des scories étant considérés comme des minéraux parmi les autres). Les problèmes abordés diffèrent sensiblement; par exemple, l'élaboration et l'utilisation du métal, d'une part et les relations minerais-scories et processus, d'autre part. Malgré tous nos efforts de compréhension, des divergences d'appréciation d'observations communes demeurent.

Il n'y a pas de méthodes capables de répondre à toutes les questions posées. Chacune possède ses spécificités, ses limites, ainsi que son domaine d'incertitudes (source d'erreurs ou de controverses), certes largement fonction de la qualité de l'échantillonnage et de l'expérience des archéomètres. Les méthodes mises en œuvre (principaux exemples dans la fig. 43) doivent être cohérentes avec les matériaux étudiés et les questions posées.

Le fer natif (météoritique ou tellurique) est anecdotique. Le fer, non métallique, existe sous deux valences chimiques: $\mathrm{Fe}^{2+}$ et $\mathrm{Fe}^{3+}$. L'atome Fer est le quatrième élément de l'écorce terrestre; il entre dans la composition de nombreux minéraux, donc de roches, et ses concentrations exploitables par l'homme, ou gisements de minerai, sont nombreuses mais de types et de dimensions très variables (Zitzmann éd., 1977-1978; Ploquin, à paraître).

Un minerai est composé de minéraux dont le fer pourra être extrait; ce sont le plus souvent des oxydes ou des carbonates, parfois des sulfures ou des silicates, et de minéraux dont le fer, s'il y en a, ne pourra pas être extrait, c'est la gangue. Celle-ci devra être éliminée, sauf si sa nature favorise l'extraction de métal; c'est l'une des fonctions des traitements préliminaires du minerai.

Les minerais à dominante sulfurée ne semblent pas avoir été utilisés significativement dans la métallurgie du fer ancienne, si ce n'est leur oxydation naturelle (les chapeaux de fer des filons ou amas sulfurés). Les minerais carbonatés, sidérose, seront transformés en oxydes par grillage avant la réduction proprement dite. Des silicates, de la famille des chamosites, participent à certains minerais (par exemple dans le faciès siliceux de la minette lorraine).

Les oxydes de fer non hydratés sont de trois types principaux:

- l'hématite, $\mathrm{Fe}_{2} \mathrm{O}_{3}$, où le fer est trivalent; c'est la forme naturelle la plus fréquente en surface, sa poussière est rouge, d'où son nom; l'oligiste, ou hématite spéculaire, en est une variante mieux cristallisée; la maghémite, de même formule, possède un réseau cristallin différent, plus proche de celui de la magnétite;

- la wustite, $\mathrm{FeO}$, où le fer est divalent; c'est la forme la plus fréquente dans les scories sidérurgiques, mais elle est exceptionnelle dans les roches terrestres;

- la magnétite, $\mathrm{Fe}_{3} \mathrm{O}_{4}$ ou $\mathrm{FeO}, \mathrm{Fe}_{2} \mathrm{O}_{3}$, associe un fer divalent à deux fers trivalents; sa structure est celle de la farnille des spinelles et $\mathrm{Fe}^{3+}$ peut être plus ou moins remplacé par $\mathrm{Al}^{3+}$; cette substitution sera fréquente et forte (transition vers l'hercynite) dans certaines scories alumineuses, sans que cela traduise des conditions plus oxydantes que dans le cas de scories, moins alumineuses, uniquement à wustite; elle est fréquente dans les roches de profondeur et peut exister dans certaines roches sédimentaires ou sous forme de placers.

Les formes hydratées les plus courantes sont celles $\mathrm{du}$ fer trivalent: la gœethite (brune) et la lépidocrocite (orangée), ainsi que les formes plus complexes de type limonite et les ocres. La forme bivalente, verdâtre et plus 
soluble, est plus rare et limitée à certains contextes confinés (marnes vertes, etc.).

Dans les carbonates, le fer est divalent mais, en cas d'altération, il forme aisément une pigmentation rouge d'oxyde de fer trivalent. Cette pigmentation rouge à brune des roches légèrement altérées est fréquente et est à l'origine de beaucoup de confusions avec des minerais, notamment en fouille.

Les traitements préliminaires du minerai à partir de son extraction du gisement (sélection, tri, lavage, concassage, grillage, lavage, débourbage, etc.) introduisent des différences qui peuvent être importantes entre le minerai " géologique " et celui qui sera effectivement utilisé.

On notera la tendance du fer à revenir à l'état d'hydroxydes, la rouille, voire de carbonates au contact de l'air humide: c'est la corrosion. Les textures et les compositions des produits de corrosion ne sont généralement pas celles des minerais (Stanton, 1972).

Le fer a une minéralogie complexe dont l'exposé sort du cadre de cette contribution ${ }^{105}$. Retenons une distinction sommaire mais nécessaire à la compréhension de ce texte:

- le fer doux contient moins de 0,02\% de carbone; sa température de fusion est de $1536^{\circ} \mathrm{C}$; il est déformable à froid, forgeable et soudable à chaud;

- l'acier contient jusqu'à $2 \%$ de carbone; sa température de fusion décroît avec cette teneur; il est plus difficile à forger et à souder mais il possède des propriétés mécaniques, dépendantes des traitements subis, qui peuvent être intéressantes: dureté, élasticité, affûtage; c'est l'art de la forge;

- la fonte contient 2 à $7 \%$ de carbone; sa température de fusion décroît encore avec cette teneur, jusqu'à $1150{ }^{\circ} \mathrm{C}$; elle peut être moulée mais non forgée (sauf procédés modernes non utilisés autrefois semble-t-il).

La différencc cntrc fcr, acier et fonte est fondamentale pour comprendre la sidérurgie. L'homme de l'art sait les distinguer par des critères visuels et mécaniques, mais le concept scientifique est récent. Les oxydes de fer, primaires (gœthite, hématite, magnétite) ou résultant du grillage d'un carbonate (ankérite, sidérite), sont " réduits " en sidérurgie traditionnelle par le monoxyde de carbone $\mathrm{CO}$ avec production de $\mathrm{CO}_{2}$. Par la suite, la

105. A. Roos, Précis de métallographie appliquée, Paris, Dunod, 1963, 456 p. et $\mathrm{H}$. de Leiris, Métaux et alliages -2- Fers, aciers, fontes, Paris, Masson, 1971, 346 p. notion de réduction a évolué de la « désoxygénation » au concept de «transfert d'électron " ${ }^{106}$.

Après avoir décrit les principaux procédés contribuant à l'élaboration du métal, nous aborderons les différents types d'approches et leurs méthodes spécifiques, puis nous expliciterons les indices de discriminations archéométriques établis ou en cours d'étude.

\section{LES PROCÉDÉS D'ÉLABORATION DU MÉTAL}

Après l'extraction minière, la préparation du minerai et son grillage éventuel, la phase de réduction (smelting) produira du fer plus ou moins carburé qui pourra être encore mélangé à des scories: il faudra l'épurer (cleansing) afin d'obtenir une masse qui pourra être mise en forme (smithing) à la forge. Si le métal produit est de la fonte (cast-iron, pig-iron) et que l'on veuille obtenir un fer forgeable (urought iron) il faudra la décarburer, c'est l'affinage (finery) (fig. 38).

Quelles que soient l'époque et la filière considérées, trois produits initiaux entrent en jeu.

Le minerai traité, caractérisé par sa composition chimique et minéralogique, sa texture et sa granulométrie. Il sera la source du métal produit mais aussi, pour l'essentiel, des scories.

Le combustible, anciennement le charbon de bois, puis le coke dès la révolution industrielle. Son rôle est multiple. Par sa combustion (réaction d'oxydation exothermique), il fournit l'élévation de température nécessaire, mais aussi, à haute température, le gaz monoxyde de carbone $\mathrm{CO}$, qui pourra piéger l'oxygène libéré par la réduction des oxydes et par la polymérisation des liquides silicatés. Sans entrer dans des considérations théoriques, disons que, en présence d'un excès de carbone, le rapport $\mathrm{CO} / \mathrm{CO}_{2}$ du gaz produit par cette combustion est d'autant plus élevé (riche en $\mathrm{CO}$ ) que la température est haute. Remarquons que des procédés modernes utilisent une autre source thermique (électricité), voire même un autre agent capteur d'oxygène

106. Cf. exposé dans N. Chezeau, P. Fluzin, Historique des aciers spéciaux, in: Béranger G., Henry G., Soulignac P. (éds), Les aciers spéciaux, Paris, Techniques et documentation Lavoisier, 1997, 1388 p. (p. 3-20). Voir aussi M. Arnold, A. Ploquin, La réduction des oxydes de fer, un autre point de vue sur la naissance du métal, Revue d'archéométrie, à paraitre. 


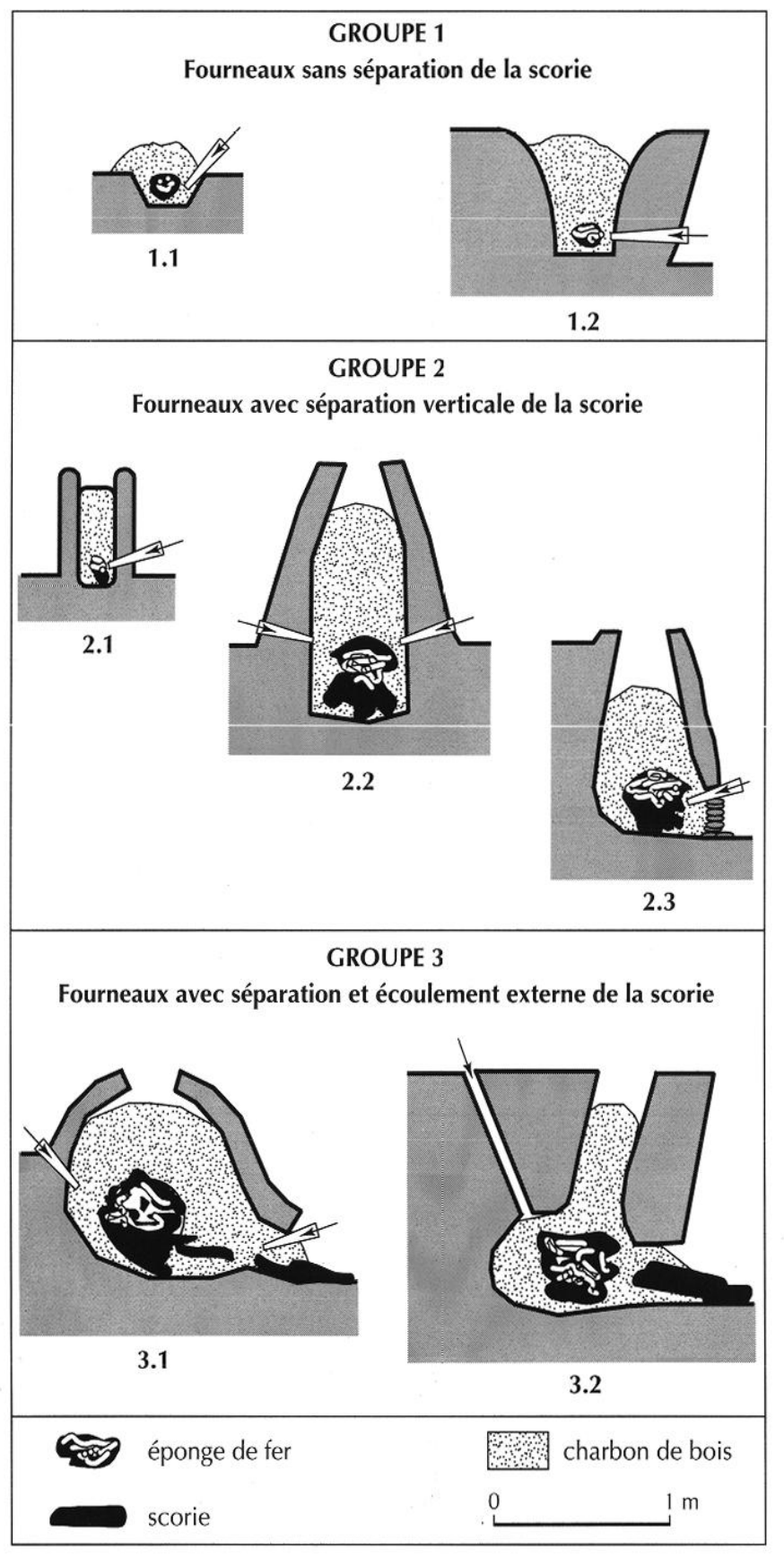

Fig. 39 - Classification simplifiée des bas fourneaux (d'après Serneels, 1998, p. 16).

(hydrogène par exemple). Le carbone a aussi un rôle dans les propriétés du métal obtenu ( $c f$. supra, p. 104), d'autres éléments peuvent également avoir de tels effets. Enfin, signalons que, par sa granulométrie et sa tenue mécanique, le charbon contribue largement à la tenue de la charge et à la circulation des gaz à travers la charge.
L'air qui contient $20 \%$ d'oxygène, élément indispensablc aux réactions de combustion du carbone, donc à l'obtention des hautes températures nécessaires. La ventilation est un facteur essentiel de la conduite des fours et conditionne le niveau des températures atteint dans les fours. Cherchant à réguler celles-ci, on abandonnera le tirage naturel pour des procédés forcés (soufflet), d'abord manuels, puis hydrauliques (dès le XII ${ }^{e}$ s.) et enfin mécaniques.

Les fours présentent de nombreuses variantes (fig. 39). Une distinction fondamentale est la température atteinte dans les fours de réduction qui conditionne l'état physique des matériaux traités.

Soit $\mathrm{T}$ la température utile requise par le procédé utilisé et Tf la température de fusion du métal:

- si T est inférieure à Tf, c'est le cas de la filière dite directe (directement du minerai au fer et/ou à l'acier). Le métal, fer et/ou acier, issu de la réduction, classiquement en bas fourneau, est encore à l'état solide ( "pâteux »); seules les scories peuvent s'écouler, soit à la base du four, soit à l'extérieur; cette filière directe (fig. 38, C-D partie gauche), malgré quelques survivances jusqu'au XIX ${ }^{e}$ s., est plutôt caractéristique de l'Antiquité et d'une grande partie du Moyen Âge en Europe et fait seule l'objet de cet article;

- si T est supérieure à Tf, nous abordons la filière indirecte; les produits issus de la réduction, classiquement en haut fourneau, sont liquides: la fonte et le laitier; bien que la fonte puisse être moulée, elle n'est pas forgeable car trop cassante et devra être décarburée pour obtenir l'acier selon deux grandes familles d'affinage: l'affinage sans fusion et l'affinage avec fusion. Cette étape supplémentaire, l'affinage, a induit l'appellation « indirect ". Cette filière indirecte (fig. 38, C-D partie droite), connue en Chine dès le VII ${ }^{e}$ s. avant J.-C. ${ }^{107}$, apparaît vers les $\mathrm{XII}^{\mathrm{e}}$ et $\mathrm{XIII}^{\mathrm{e}}$ s. en Allemagne (comté de Mark, Nordrhein-Westfalen) ${ }^{108}$ et

107. J. Needham, The Evolution of Iron and Steel Technology in East and Southeast Asia, in: Wertime T. A., Muhly J. D. (eds), The Coming of the Age of Iron, London, Yale University Press, 1980, p. 507-54l et Hua Jue-Ming, Les moulages à métaux ferreux dans l'ancienne Chine, Pour la Science, mars 1993, p. 66-73.

108. I. Knau, D. Horstmann, M. Sonnecken, La production de fonte dans la haute vallée de la Volme: contribution à l'histoire de la sidérurgie en Westphalie occidentale, in: L'innovation technique au Moyen $\hat{\Lambda} g e, \Lambda$ ctcs du $\mathrm{VI}^{\mathrm{c}}$ congrès international d'archéologie médiévale, Paris, éd. Errance, 1998, p. 152-159. 

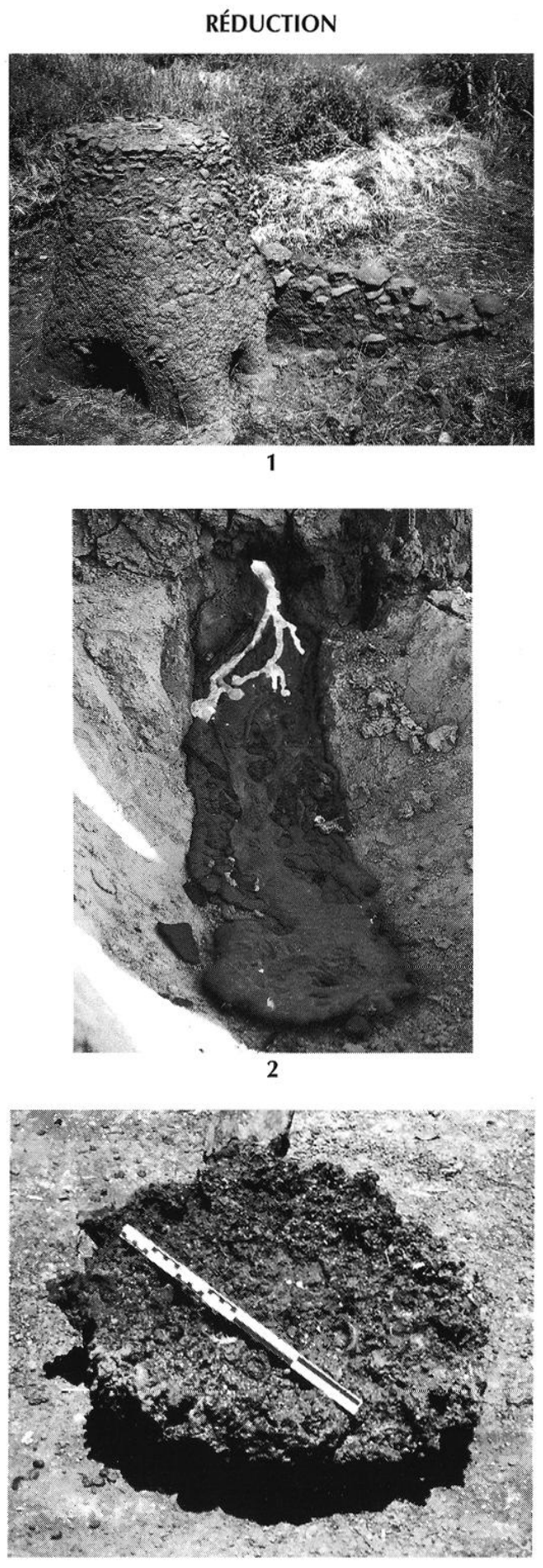

3

Fig. 40 - 1, bas fourneau contemporain, sile de Toungaré, province du Bulkiemdé, Burkina Faso (cliché P. Fluzin, 1994); 2, scories denses coulées en plaque (cordons successifs), bas fourneau de type Aunay-Truchet, Sarthe (expérimentation: 1997, C. Dunikowski, S. Cabboï, P. Fluzin, A. Ploquin; cliché P. Fluzin); 3, loupe comtemporaine de $60 \mathrm{~kg}$, site de Toungaré, province du Bulkiemdé, Burkina Faso (cliché P. Fluzin, 1994). en Suède ${ }^{109}$ et se généralisera au XVI ${ }^{e}$ s. Cependant, des exemples archéologiques montrent que la fonte fut aussi produite dans des bas fourneaux de façon accidentelle (Pelet, 1993; Yalcin, Hauptmann, 1995), ou volontaire (Mahé-Le Carlier et al., 1998).

L'instrument de fabrication de la fonte est normalement le haut fourneau, mais l'on doit mentionner des découvertes très récentes de sites à bas fourneaux:

- en Suisse et en Allemagne, où des coulures de fonte ont été trouvées; cette fonte est considérée comme accidentelle (Pelet, 1993; Yalcin, Hauptmann, 1995);

- en Italie (Val Camonica, Val Gabbia), où l'on a produit aux Ve-VIe $\mathrm{s}$. après J.C. des blocs de fonte blanche de $3,5 \mathrm{~kg}$ qui ont été décarburés (Fluzin, 1999);

- en France (Oulches, Berry), où une fosse permettait "d'étonner » des blocs de laitiers à grenaille obtenus en modifiant la charge des gros bas fourneaux par un ajout de calcaire; ce traitement, qui fut utilisé au IV s. après J.C., permettait de récupérer une grenaille à diverses teneurs en carbone (Mahé-Le Carlier et al., 1998) ; cependant sur ces sites l'essentiel de la production est de type « filière directe ";

- en France et en Suisse, bas fourneaux dont les scories présentent des caractères mixtes (cf. infra, type 3, p. 116).

\section{LA CHAÎNE OPÉRATOIRE EN SIDÉRURGIE DIRECTE}

En sidérurgie directe nous distinguerons trois étapes principales (réduction, épuration, mise en forme de l'objet) qui sont ou non associées dans l'espace (fig. 38, partie gauche). Chacune implique des procédés techniques et génère un produit et des déchets plus ou moins spécifiques. Nous les résumerons sommairement sans entrer dans la description des processus physicochimiques.

\section{LA RÉDUCTION DIRECTE}

Cette étape consiste à transformer le minerai de fer en métal brut dans un bas fourneau (fig. $40, \mathrm{n}^{\circ} 1$ ). Le fer

109. G. Magnusson, I apphyttan, in: Sperl G. (éd.), The Fïrst Irom in the Mediterranean, Symposium Populonia/Piombino 1983, PNCT, 21, Strasbourg, 1988, p. 177-198. 
ou l'acier ainsi produits ne seront pas liquides en masse: il n'y a pas de coulée de métal. La gangue du minerai et divers produits (cendres du charbon de bois, une partie du revêtement interne et parfois des ajouts) se combinent à une part importante des oxydes de fer et forment des scories de forte densité, abstraction faite de leur porosité (fig. $40, n^{\circ} 2$ ). Il serait trop long de faire une typologie précise des bas fourneaux, tant leur diversité est grande, mais il est possible de les regrouper, par exemple, en trois familles selon que la scorie se sépare plus ou moins facilement du métal, qu'elle s'écoule à l'intérieur, ou à l'extérieur du fourneau (fig. 39) (Cleere, 1972; Pelet, 1982; Tylecote, 1987; Bielenin et al., 1998; Serneels, 1998).

Dans la plupart des types de bas fourneau (fig. 39), on introduit dans la partie supérieure, alternativement, du charbon de bois et du minerai ou des mélanges de minerais et éventuellement des ajouts. La combustion est assurée par une ventilation naturelle et/ou forcée à l'aide de soufflets.

À l'issue de la réduction (dont la durée varie avec le type de fourneau et les conditions de ventilation: elle peut, selon nos expérimentations et les relations ethnographiques, aller de 6 à 56 heures), on retire du fourneau, par une ouverture ou en détruisant le four, une masse (massiot). C'est un agglomérat, plus ou moins compact, de métal (fer-acier), de scories et de charbon de bois. Selon la compacité du métal on parle d'éponge de fer ou de loupe (fig. $40, \mathrm{n}^{\circ} 3$ ). Celle-ci peut peser, suivant la taille du four et la quantité de minerai introduit, de quelques centaines de grammes à plus de $100 \mathrm{~kg}$, notamment pour des exemples africains que nous connaissons ${ }^{110}$, voire même à plusieurs centaines de kilogrammes dans des procédés tardifs (Stückofen, four catalan).

La maîtrise de la conduite du procédé influence beaucoup la qualité et la séparation du métal et des scories qui est rarement suffisante pour ne pas exiger un travail d'épuration. Il est probable que, dans presque tous les cas, ce travail était indispensable.

L'examen visuel des morphologies des scories apporte des informations sur le fonctionnement et

110. G. Celis, Les fonderies africaines du fer, un grand métier disparu, Frankfurt am Main, 1991, 225 p. et V. Serneels, E. Huysecom, P. Fluzin, Inagina iron-slags and cutcctoïd stccl, in: Crew P., Crew S. (eds), Early Ironworking in Europe. Archaeology and Experiment, vol. 2, à paraître. l'architecture du fourneau (Serneels, 1993; Doswald et al., 1997; Leroy, 1997; Leroy, Merluzzo, 1998). On distingue principalement des scories coulées à l'extérieur du fourneau sous forme de plaques ou de cordons simples ou superposés. Elles peuvent mouler une dépression ou un chenal aménagé devant le four. Celles qui restent dans le fourneau se présentent sous forme de blocs plus ou moins compacts moulant le fond de la cuve, imbriqués dans la masse de charbon de bois ou percolant au travers de matériaux disposés dans une fosse aménagée sous le four. De même, l'examen des vestiges de four apportera des renseignements (Merluzzo, Leroy, 1999). Nous sommes là à la frontière entre archéologie et archéométrie.

Ces déchets forment l'essentiel des accumulations qui seront les premiers indices repérables en prospection. Un petit ferrier dépassera fréquemment une tonne de scories; les ferriers de plus de 10000 t ne sont pas rares.

\section{L'ÉPURATION}

L'épuration consiste en un martelage à chaud (cinglage) de la loupe afin de rassembler (grapper) le métal en évacuant les impuretés et les vides. Le travail se fait à partir de la masse, directement à la sortie du four ou après réchauffage dans un foyer ou four spécifique (fig. 41, $\mathrm{n}^{\mathrm{os}} 1$ et 2) (Fluzin et al., 1995), mais il n'est pas exclu de réutiliser le bas fourneau pour ce type d'opération; la loupe est introduite telle quelle ou par gros fragments afin de fluidifier au maximum les scories associées, notamment dans le cas de loupe "sale ". Certains exemples ethnologiques et archéologiques démontrent cette pratique. L'éponge de fer (loupe) est ainsi « asséchée " plus ou moins efficacement de ses scories externes et internes. Durant cette opération la carburation du métal peut se renforcer (cémentation). On peut ainsi obtenir des aciers très carburés, voire de la fonte, en traitant plusieurs fois la loupe dans le même four.

L'opération d'épuration peut comporter plusieurs étapes (dégrossissage primaire, puis secondaire). Les déchets associés peuvent être des fragments de scories coulées, des scories informes plus ou moins riches en métal, des fragments métalliques déchiquetés imbibés de scories; ces derniers ont été dénommés gromps (Nosek, 1994). Ils sont caractéristiques de ce nettoyage et compactage de la loupe; leur proportion varie considé- 
rablement d'une loupe à l'autre en fonction de la qualité de celle-ci (densité du métal, propreté inclusionaire, porosité, etc.). Divers matériaux fondus s'accumulent au fond ou en bordure du foyer d'épuration et forment une scorie en forme de calotte (ou culot) plus ou moins hémisphérique ou plano-convexe. Ces calottes d'épuration sont généralement de taille importante et hétérogènes avec de nombreuses inclusions de fragments métalliques (fig. $41, \mathrm{n}^{\circ} 2$ ). Lorsque l'on utilise des ajouts « désoxydants » ou «fondants » (sable, argile, cendres de graminées, etc.), la scorie produite sera plus siliceuse, parfois plus alcaline, souvent vitreuse, et pourra former soit des gouttes, soit des portions plus ou moins nettes de calottes.

La quantité de scories d'épuration d'une loupe "propre » ou d'une épuration secondaire peut être très faible et échapper à l'observation archéologique. Mais, d'après nos expérimentations et l'ethnoarchéologie, il s'avère que l'épuration d'une loupe considérée comme «sale » occasionne des pertes d'environ $80 \%$ du poids initial avant qu'on obtienne un lingot ${ }^{111}$. Ces pertes se répartissent entre $50 \%$ d'éléments à forte teneur en métal déchiqueté enrobés de scories (cf. gromps), $10 \%$ de battitures et $20 \%$ de scories et billes. Le poids de la calotte peut atteindre la moitié de celui de la loupe initiale. Sur un site de réduction, les déchets attribuables à l'épuration sont généralement sous-estimés, voire non décelés.

Le produit issu de l'épuration est en principe un demi-produit (lingot) plus ou moins bien manufacturé comme en témoignent quelques exemples archéologiques, ethnologiques ou de reconstitution. La mise en forme du lingot (fig. $41, \mathrm{n}^{\circ} 3$ ), compte tenu des efforts qu'elle nécessite, doit traduire la spécialisation d'un atelier ou une destination particulière à caractère " commercial» (cas des lingots bipyramidaux ou des currency bars, cf. Crew, 1994). En effet, les exemples africains nous indiquent que pour une utilisation locale on ne s'embarrasse pas à produire des formes particulièrement élaborées (Fluzin et al., 1995). Ces lingots peuvent prendre l'aspect de fragments de loupe sommairement

111. P. Crew, The Experimental Production of Prehistoric Bar Iron, Historical Metallurgy, 25, 12, 1991, extrait 16 p. et P. Dillmann, P. Fluzin, M. Urtega, Refining of an experimental Biscaian bloom from Agorregi, in: Crew P., Crew S. (eds), Early Ironworking in Europe. Archaeology and Experiment, vol. 2, à paraître.
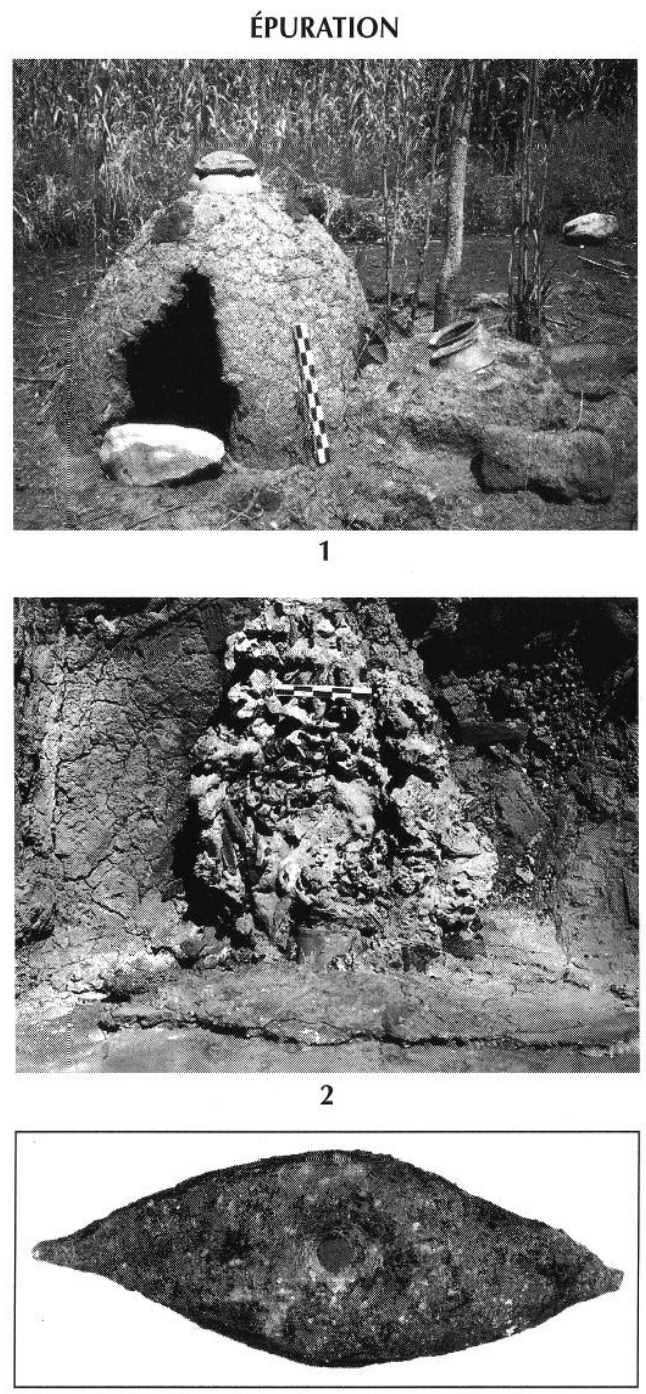

3

Fig. 41 - 1, foyer de forge d'épuration contemporain, site de Toungaré, province du Bulkiemdé, Burkina Faso (cliché P. Fluzin, 1994); 2, foyer de forge et culot après épuration d'une loupe de $4,7 \mathrm{~kg}$, poids du culot avec argile: $1,5 \mathrm{~kg}$ (expérimentation: Nancy, 1997, D. Leclère, P. Fluzin, M. Leroy, P. Merluzzo; cliché P. Fluzin); 3, lingot provenant de Carthage (IVt-III s. avant J.-C.), poids $1,77 \mathrm{~kg}$, longueur $20 \mathrm{~cm}$, largeur $6,5 \mathrm{~cm}$, épaisseur $4 \mathrm{~cm}$ (cliché P. Fluzin).

épurée, de barres, de feuilles repliées, de "saumons ", de bipyramides effilées, d'ébauches, de currency bars, de « doigts » associés, etc., de taille variable.

Signalons une autre manière de procéder à une épuration, pratiquée notamment par des artisans japonais: fragmentation à froid de la loupe afin de trier le fer et l'acier sous le marteau, puis assemblage en lingots différents par soudure, à la forge sur une palette. 


\section{LE FORGEAGE DE L'OBJET}

Cette phase est pratiquée par le forgeron sur l'enclume pour mettre en forme les objets de fer. L'emploi du terme forgeage devrait être limité aux
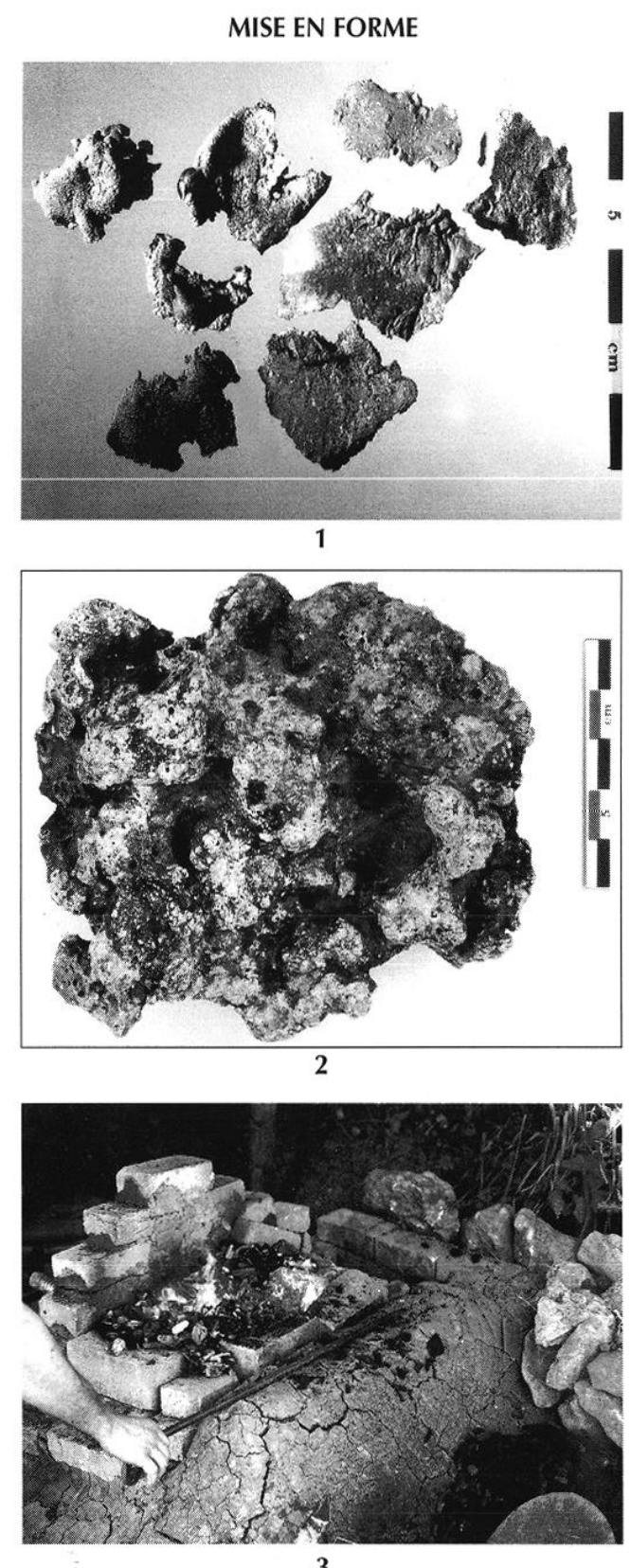

3

Fig. 42 - 1, battitures produites lors de la forge d'un objet (expérimentation: D. Leclère, P. Fluzin; cliché P. Fluzin); 2, culot de forge gallo-romain (Saverne, Haut-Rhin), poids $205 \mathrm{~g}$, vue de dessus (A.-M. Adam, P. Fluzin; cliché P. Fluzin); 3, foyer de forge (expérimentation: Nancy, 1997, D. Leclère, P. Fluzin, M. Leroy, P. Merluzzo; cliché P. Fluzin). traitements thermomécaniques de mise en forme des objets, et aux traitements thermochimiques qui modifient la structure et la composition du métal. Le matériau de départ peut être un lingot ou une ébauche de l'objet à produire ou un lopin issu du recyclage de différents fragments métalliques. Les déchets les plus caractéristiques de cette étape sont les battitures (fig. $42, \mathrm{n}^{\circ} 1$ ). Elles sont plus abondantes à proximité de l'enclume. Les diverses matières fondues peuvent s'accumuler dans le foyer et former une scorie en forme de calotte (fig. 42, $\mathrm{n}^{\circ}$ 2). Fréquemment, les " désoxydants " ou « fondants" apportent une contribution qui peut être importante à la formation des culots de forge, notamment dans le cas de soudures. On trouve aussi des scories informes plus ou moins riches en métal. En principe, ce travail ne produit pas de scories coulées; si elles existent, elles sont en petites quantités.

Il est important de rechercher les chutes provenant de ce travail; elles sont généralement très rouillées, voire minéralisées, et ne sont que trop rarement prises en compte lors de la fouille. Ce sont des indices des travaux effectués: découpes, soies de préhensions, métal brûlé, etc.

Les quantités de déchets trouvés sur les sites de forge sont généralement réduites: quelques dizaines de kilogrammes, rarement plus de $1 \mathrm{t}$ (Dunikowski et al., 1996).

On conçoit que, si les déchets issus de la réduction et ceux produits par les opérations qui suivent (épuration, mise en forme) peuvent être aisément distingués, la distinction des calottes et des battitures issues des deux phases postérieures à la réduction est plus difficile, voire impossible. De plus ces deux phases peuvent se pratiquer dans le même atelier et utiliser les mêmes foyers ou des foyers analogues (fig. $42, n^{\circ} 3$ ). À la limite elles peuvent ne constituer qu'une seule opération; le massiot, ébarbé de ses parties hétérogènes, est épuré par cinglage et immédiatement transformé en lingot puis éventuellement en objet, dans le même cadre spatial, avec les mêmes structures et les mêmes outils.

\section{LES MÉTHODES ARCHÉOMÉTRIQUES APPLIQUÉES À L'ÉTUDE DES VESTIGES SIDÉRURGIQUES ANCIENS}

Les méthodes d'investigation des matériaux en laboratoire sont nombreuses (fig. 43). Cependant, les résultats de ces examens, relativement lourds et coûteux, 


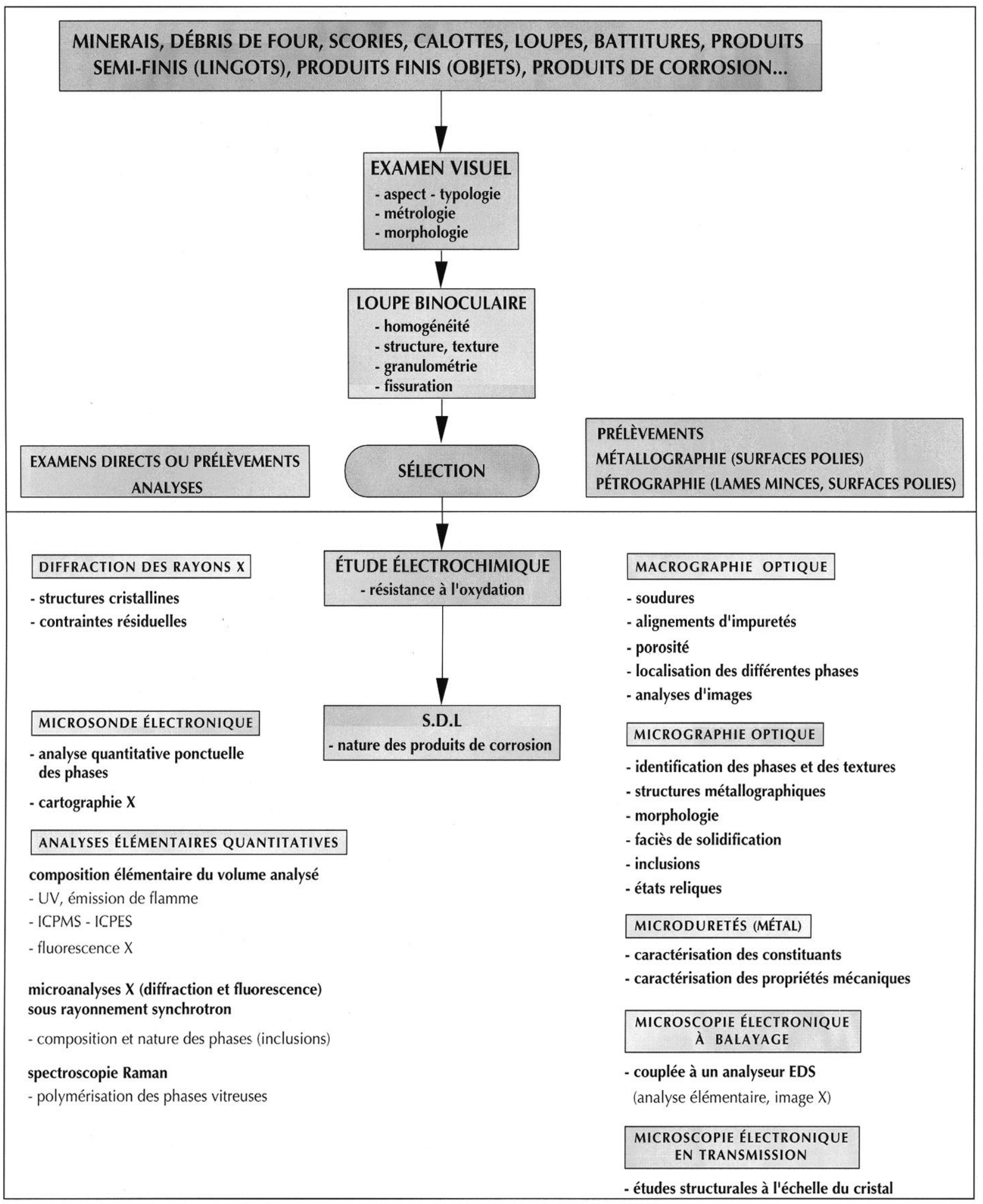

Fig. 43 - Études à caractère métallurgique susceptibles d'être utilisées. 
dépendent étroitement de la qualité de l'échantillonnage et des autres données fournies par les constats archéologiques de terrain. Lors de la fouille d'ateliers, la répartition spatiale de certains déchets permet d'identifier des aires de travail spécialisées (minerai non réduit dans les aires de chargement des bas fourneaux, battitures autour d'une enclume, etc.).

En plus de l'étude des structures et des relations stratigraphiques, il est essentiel de prendre en compte l'ensemble des déchets métallurgiques (typologie, répartition, quantification). C'est une condition indispensable à la réalisation d'un échantillonnage cohérent. Par ailleurs, il convient de ne pas oublier l'éventuelle présence de déchets pouvant correspondre à des échecs, des essais, des changements techniques, ou même à des apports exotiques.

L'étude est compliquée par l'hétérogénéité de la physico-chimie des matériaux résultant de la métallurgie du fer en phase solide. La cinétique des procédés mis en œuvre est en général très loin d'atteindre les conditions d'équilibre théorique (gradients de température et des pressions partielles dans les fourneaux, irrégularités dans les flux gazeux, etc.). Quelles que soient les méthodes d'investigation, l'observation devra être effectuée sur des prélèvements suffisamment grands pour être significatifs. Constamment il faudra être en mesure de relier les observations faites à diverses échelles.

Enfin, les hypothèses élaborées sur les matériaux archéologiques et sur la gestuelle technique peuvent être validées en comparant ces matériaux aux déchets et produits des reconstitutions expérimentales et à ceux qui proviennent des procédés traditionnels, tels que l'on peut encore les observer. L'ethnoarchéologie fournit aussi des idées concernant les aspects sociaux et économiques: transmission des savoir-faire, organisation du travail et des échanges, etc.

L'étude des déchets métallurgiques porte essentiellement, outre la morphologie macroscopique, sur l'identification des constituants (chimie et minéralogie) et l'observation des textures microscopiques qui témoignent du mode de formation de ces matériaux.

\section{LA MÉTALLOGRAPHIE}

(Fluzin, 1994)

Son apport majeur est l'identification des phases métalliques et l'observation des textures microscopi- ques: l'aspect des grains métalliques et oxydes, les inclusions; leurs relations mutuelles et avec leur environnement, matrice silicatée. L'échantillon est scié et la surface plane obtenue est finement polie. L'étude se fait alors avec un microscope métallographique (lumière réfléchie) qui permet d'observer l'aspect, la disposition et la forme des grains de métal en utilisant le fort pouvoir réflecteur de ces matériaux. L'utilisation de différents dispositifs et réactifs facilite l'observation. La dureté du matériau peut être mesurée par poinçonnement ponctuel. Des sections polies de grandes dimensions (supérieures à $100 \mathrm{~cm}^{2}$ ) peuvent être obtenues.

\section{LA PÉTROGRAPHIE}

(Jarrier, 1993; Ploquin, 1994a; Mahé-Le Carlier, 1997)

Son premier outil est le microscope pétrographique. Il permet d'étudier les autres composants, silicates, oxydes et verres principalement. On prélève une partie de l'échantillon pour obtenir par rodage une lame de $30 \mu \mathrm{m}$ d'épaisseur ; ce travail est généralement plus difficile que dans le cas de roches normales. L'observation, en lumière transmise, porte sur les textures et l'identification des phases à l'aide des interactions entre la lumière polarisée ou non et les matériaux transparents à cette épaisseur (silicates, verres, etc.). De préférence la surface de la lame mince est polie, de manière à permettre, comme avec des sections polies, l'observation en lumière réfléchie des cristaux opaques (métaux, oxydes, etc.). Le format standard de ces lames est de $25 \mathrm{~mm}$ x $37 \mathrm{~mm}$; des lames plus grandes peuvent être réalisées afin d'avoir une meilleure continuité d'observation mais cela pose des problèmes de tenue et de régularité et elles ne pourront pas être utilisées dans les microsondes. Les lames contenant du métal sont sensibles à la corrosion.

La diffraction des rayons $X$ permet d'identifier les phases cristallisées (via leurs distances interréticulaires) présentes dans un prélèvement de quelques millimètres cubes réduit en poudre.

\section{LA CHIMIE}

(Ploquin, 1994a; Mahé-Le Carlier, 1997)

Les microsondes, via la réponse à l'impact d'un faisceau de quelques microns de diamètre, donnent des 
teneurs chimiques «ponctuelles» de la cible. Les variations relatives d'une teneur peuvent être cartographiées sur une petite surface (scanning). Ce travail se fait sur une surface très bien polie (lame mince ou section). L'usage d'étalons ad hoc est indispensable.

Le microscope électronique à balayage (MEB) permet de visualiser une surface (cassure fraîche) et d'en donner une composition chimique qualitative.

\section{L'ANALYSE CHIMIQUE GLOBALE}

(La Roche, 1972; Ploquin, 1992, 1993; Serneels, 1993, 1994; Mangin et al., 1994)

Ses résultats permettent une réflexion sur la composition des matières premières mises en oeuvre et leurs transformations. L'échantillon est réduit en poudre; le choix du prélèvement doit donc être effectué avec toute la rigueur nécessaire pour en assurer la représentativité. Différentes méthodes chimiques ou physico-chimiques (XRF, ICP, etc.) permettent ensuite de mesurer les concentrations des éléments présents dans la poudre. La préparation (dilution dans un verre, poudre pressée, dissolution par attaque chimique, etc.) varie en fonction de la méthode d'analyse, de même que le nombre des éléments dosés et la précision des mesures. Il faut privilégier les procédures qui utilisent des étalons reconnus.

D'autres techniques d'analyse peuvent être utilisées (spectroscopie Raman, effet Mosbauer, etc.). Citons deux exemples prometteurs d'utilisation de techniques inhabituelles dans notre domaine. La spectroscopie Raman a permis d'aborder la polymérisation des verres de scories et son rôle dans leur altérabilité (Mahé-Le Carlier, 1997) et dans la réduction ${ }^{112}$. La microdiffraction et la microfluorescence $\mathrm{X} d \mathrm{du}$ rayonnement synchrotron a permis d'initier l'étude des inclusions de scories dans le métal et ainsi d'aborder le difficile problème de l'identification de l'origine technique d'une masse de métal ${ }^{113}$. Ce dernier

112. A. Ploquin, M. Arnold, C. Mahé-Le Carlier, What part play electron transfert and polymerisation of silicate melt in iron-making process?, à paraître (cf. résumé, CSA Jutland conference, sept. 1999).

113. P. Dillmann, Diffraction $X$, microfuorescence $X$ sous rayonnement synchrotron el analyses comparées pour la caractérisation des inclusions. Applications à l'élude de l'évolution historique des procédés d'élaboration des objets ferreux, thèse de Doctorat, univ. de Compiègne, 1998, 300 p. ; P. Dillmann, P. Fluzin, P. Benoit, Du fer à la fonte. Nouvelles approches archéométriques, in: Linnovation technique au Moyen Âge, Actes du VI" congrès international de la Socićtć d'archćologic mćdićvialc, Dijon, oct. 1996, Paris, éd. Errance, 1998, p. 160-168; P. Dillmann, P. Populus, développement, issu d'une équipe "métallographique ", prend une orientation de type «pétrologique ».

\section{INDICES DE DISCRIMINATION}

L'objectif est de préciser un certain nombre d'indices discriminatoires de la chaîne opératoire pour tenter de cerner ce qui se passe lors de l'élaboration du métal (du minerai à la loupe) et de la mise en forme d'un objet. Il importe de préciser que si la présence d'un indice peut être significative d'une opération, son absence est une information précieuse mais non obligatoirement significative d'une non-opération: il peut avoir été "effacé ", notamment par une élévation de température. C'est l'analyse de cohérence de la présence et/ou de l'absence des différents indices et de leur contexte qui permet d'élaborer les hypothèses les plus fiables.

\section{APPROCHE DE TYPE PÉTROLOGIQUE}

Minerais plus ou moins traités, parois de four, scories de différents types et autres produits associés sont des matériaux minéraux qui peuvent être considérés comme des roches anthropiques. La démarche pétrologique peut leur être appliquée, avec les mêmes outils. Cependant quelques différences de fond existent et impliquent une certaine reconversion de la mise en œuvre. Les compositions des scories sont très rarement analogues à celles des roches: les scories sont généralement beaucoup plus riches en Fe (réduction en bas fourneau, forge, affinage) et moins alcalines. Les relations entre les divers éléments n'obéissent pas tout à fait aux mêmes "logiques". Les conditions de genèse sont différentes de celles des roches volcaniques: températures souvent supérieures, pressions partielles d' $\mathrm{O}_{2}$ ou

P. Fluzin, P. Chevallier, E. El Kaim, G. Béranger, Microdiffraction du rayonnement synchrotron. Identification de phases non métalliques dans les fers anciens, La Revue de métallurgie, CIT/Sciences et génie des malériaux, fév. 1997, p. 267-268; P. Dillmann, P. Populus, P. Chevallier, E. El Kaim, P. Fluzin, G. Béranger, A. Firsov, Microdiffraction du rayonnement synchrotron. Identification de phases non métalliques dans les alliages ferreux, Compte rendu de l'Académie des Sciences/Elsevier, 324, IIb, Paris, 1997, p. 763-772; P. Dillmann, P. Populus, P. Chevallier, P. Fluzin, G. Béranger, A. Firsov, Microdiffraction coupled with X-Ray Fluorescence microprobe, application in the archaeometry, Journal of trace and microprobe techniques, 15 (3), 1997, p. 251-262. 
d' $\mathrm{H}_{2} \mathrm{O}$ très basses, pression partielle de $\mathrm{CO}$ très forte, très fort rapport $\mathrm{CO} / \mathrm{CO}_{2}$, pression totale peu supérieure à $1 \mathrm{~atm}$, gradients de température importants, cinétique forte. Il en découle que les minéraux formés sont de nature marginale ou différente de ceux qui sont dans les roches; mais surtout leurs morphologies sont très rares dans la nature (ex. texture spinifex de certaines laves basiques).

Pour des raisons non seulement de rapidité de mise en œuvre mais aussi de pertinence, nous avions donné priorité à l'approche géochimique de la réduction (Ploquin, 1994a). L'un des premiers résultats marquants fut la démonstration de l'utilisation de la minette lorraine en bas fourneaux (Leroy et al., 1990; Leroy, 1997). Progressivement des études pétrographiques ont été développées, puis connurent une certaine accélération, notamment avec les thèses de $\mathrm{C}$. Jarrier (scories de bas fourneaux, cf. Jarrier, 1993), M. Leroy (scories de bas fourneaux, minerais en cours de réduction, $c f$. Leroy $e t$ al., 1994 et Leroy, 1997) et C. Mahé-L.e Carlier (scories de bas et haut fourneaux anciens, cf. Mahé-Le Carlier, 1997); des scories et battitures de forges (Bailly-Maître, Ploquin, 1993; Dunikowski et al., 1996), des scories d'affinage, des scories de métallurgie au creuset, des minerais partiellement réduits ont été, et sont, également étudiés. Les spécimens étudiés sont surtout d'origine archéologique, mais proviennent aussi de reconstitutions expérimentales. Tout ceci forme maintenant un corpus et correspond désormais à un savoir-faire important, savoir-faire malheureusement partagé par trop peu de personnes.

Nous allons présenter d'abord les outils puis certaines modalités d'interprétation.

\section{LES OUTILS}

N'oublions pas l'œil. Aidé de la loupe, éduqué par l'expérience, il permet de remarquer beaucoup de choses sur les scories: morphologie, faciès pétrographique, traces d'outils, inclusions, typologie pouvant être complétée par des pesées ou estimations, etc.; sur les parois: zonation, gradients de température, traces de fusion, etc.; sur les minerais: variations, traces de traitements, etc. Mais surtout, même sans comptage, il permet généralement de distinguer, sur place, ce qui est " normal » de ce qui est "particulier ", de procéder à une première sélection des spécimens à étudier (voir « échan- tillonnage », in: Ploquin, 1992, 1994a). Le sciage, à la scie diamantée avec refroidissement, suivi d'un dressage, permet de prolonger cet examen sur des coupes: relation entre faciès, inclusions, métal, porosité. Notons qu'une surface sciée dressée et une surface cassée n'ont pas la même lisibilité, variable d'un cas à l'autre.

L'usage des techniques de laboratoire sera fonction de l'étude à réaliser et des moyens disponibles.

L'observation au microscope, en transmission et en réflexion, permet de détecter et, souvent, de reconnaître les différentes phases cristallisées (minéraux) ou non (verres, gels), d'observer leurs morphologies et leurs relations mutuelles. Il est fondamental de bien repérer la lame par rapport au spécimen. Les rayons $\mathrm{X}$, via diffractogrammes sur poudre, apportent une aide à la détermination des phases cristallisées suffisamment abondantes. Sur lames minces polies ou sections polies, les microsondes électroniques permettent de préciser, ponctuellement (i.e. quelques $\mu \mathrm{m}$ ) la composition chimique (éléments majeurs ou mineurs); ce type d'analyse demande une bonne connaissance préalable de la lame ou section et un repérage des points à « viser »; certaines variantes permettent de cartographier un élément sur une petite surface. La microsonde ionique, d'usage encore très limité, permet d'atteindre des éléments en traces, voire leurs isotopes.

L'analyse chimique globale s'applique à un volume plus grand et livre les teneurs des éléments dosés correspondant à ce volume. En fait, la prise qui sera effectivement analysée est minime (en ICP-ES ou MS la prise est de l'ordre de $300 \mathrm{mg}$ ), mais cette prise est prélevée sur une quantité plus importante de poudre homogénéisée ou de solution afin d'éviter les effets de grain ou de pépite. On conçoit donc que le choix et la préparation de l'échantillon soient primordiaux: tout ce qui sera broyé ou mis en solution participera aux nombres (teneurs) fournis par la machine. Actuellement les diverses méthodes analytiques sont fiables, sous réserve que le laboratoire se contrôle constamment par l'usage d'étalons reconnus. Le choix est alors plutôt celui des listes d'éléments dosés dans les gammes de teneurs adaptées.

C'est l'expérience du pétrographe-géochimiste qui décèlera les cohérences-incohérences entre ces diverses observations et mesures. Il importe de toujours conserver un témoin de l'échantillon étudié afin de pouvoir revenir à l'examen direct. 


\section{LES MOYENS D'INTERPRÉTATION}

\section{Principes de base relatifs aux compositions}

La composition chimique d'une quantité de matière est égale à la somme pondérée des compositions de ses phases constitutives, ou " règle du barycentre " (La Roche, 1972). Cette règle est fondamentale pour tout traitement graphique des résultats d'analyses à l'aide de diagrammes, ou pour tout calcul de bilan (Ploquin, 1992, 1993, 1994a; Serneels, 1993; Leroy, 1997).

Lors d'une transformation en système clos " rien ne se perd, rien ne se crée, tout se transforme ", ainsi par exemple, la fusion d'une charge. Il reste à bien définir quel est le «système ». Dans le cas d'un four, l'air injecté et les fumées évacuées font partie du système pour un bilan global; selon l'élément considéré il faudra en tenir compte (par exemple, $\mathrm{Zn}$, As, $\mathrm{CO}, \mathrm{CO}_{2}$, etc. volatils) ou non. La ségrégation d'une phase, par exemple le métal qui se sépare de la scorie, peut être considérée comme une sortie du système « scorie ". Un apport de " décapant » dans une forge est un ajout au système, il entrera dans la composition de certains déchets (scories, battitures).

Lors d'une transformation drastique (par exemple, fusion et solidification), les éléments se redistribuent selon leurs affinités; mais, et c'est une différence importante par rapport au comportement géologique, cette redistribution pourra ne pas être aussi réglée que les lois de la thermodynamique le disent, du fait de la rapidité des réactions de cristallisation; ainsi les équilibres ne sont pas toujours atteints et les « coefficients de partage " d'un élément entre plusieurs phases ne seront pas toujours cohérents. Ce comportement peut varier selon les conditions (par exemple, $\mathrm{P}, \mathrm{V}, \mathrm{Fe}$ en bas ou haut fournéau). Les éléments qui se «logeront» de préférence dans le fer métal (comportement sidérophile, par exemple, $\mathrm{Ni}$ ) ou dans la scorie (comportement lithophile, par exemple, Be, Y, Th, les Terres Rares) constitueront s'ils sont suffisamment abondants, par exemple dans le minerai, des marqueurs hérités du minerai. Les connaissances des géologues aident à définir ces marqueurs, ainsi par exemple, une corrélation $\mathrm{Al}, \mathrm{V}, \mathrm{Cr}$, Ti est un bon indicateur d'une phase argileuse dans les matériaux, et $\mathrm{Si}, \mathrm{Zr}$ d'une phase gréseuse. Ces phases argileuses ou gréseuses peuvent provenir de la gangue du minerai ou de la paroi du four ou des cendres du charbon. $\mathrm{Ca}, \mathrm{Si}, \mathrm{Mn}, \mathrm{K}, \mathrm{P}$ peuvent provenir, pro parte, des cendres du charbon de bois selon l'origine du bois et cet apport des cendres n'est pas insignifiant ${ }^{114}$. C'est la règle de "l'héritage et des coefficients de partage ». Pour chaque cas des diagrammes adaptés permettront de discuter. Donnons quelques exemples d'utilisation de cette notion d'héritage ou traçage chimique du minerai:

- la démonstration de l'utilisation de la minette lorraine en bas fourneau, à contre-courant de l'opinion générale (Leroy et al., 1990, 1997; Leroy, 1997);

- la démonstration de l'utilisation d'ajout calcaire lors $\mathrm{du}$ « fonctionnement en régime haut fourneau » du bas fourneau antique d'Oulches (Mahé-Le Carlier et al., 1998);

- des calculs de bilans et de rendements d'opérations (Serneels, 1993; Leroy, 1997).

Cette notion s'applique aussi à la distinction de scorie fayalitique de réduction et du faciès fayalitique de scorie de forge ( $c f$. faciès b, in: Dunikowski et al., 1996). Les éléments qui constituent la scorie de forge ont plusieurs origines: le minerai, mais uniquement les éléments qui auront pu transiter par le métal (éléments sidérophiles) ou via les inclusions de scories dans le métal (donc plus sensibles dans le cas de l'épuration, surtout primaire), les ajouts ( $c f$. décapants) et les cendres du charbon. La participation des ajouts sera plus évidente si leur incorporation dans la calotte n'est pas totale ( $c f$. faciès a, in: Dunikowski et al., 1996).

Enfin, dans des conditions physico-chimiques données (température, pressions partielles de $\mathrm{CO}$, d'O $\mathrm{O}_{2}$, compositions relatives, etc.), c'est une certaine composition minéralogique qui est susceptible de se former aux dépens des matériaux présents. Heureusement, une baisse rapide de la température peut « bloquer » cette association minérale, que nous pourrons alors observer sauf « altérations secondaires », c'est-à-dire les évolutions vers des équilibres avec un milieu notamment plus oxydant, hydraté et froid, le nôtre ou celui du site archéologique. Des diagrammes, dits de phases, donnent ces

114. Maintenant nous pouvons analyser le charbon de bois comme les autres matériaux, mais l'exigence de propreté est encore plus contraignante. Si le taux de cendre est de $5 \%$, une pollution du charbon par 1 ou $2 \%$ de terre sera catastrophique; certains éléments, s'ils sont présents dans les eaux vadoses, se fixeront sur le charbon ( $\mathrm{Ba}, \mathrm{As}, \mathrm{Sr}$ par exemple). 


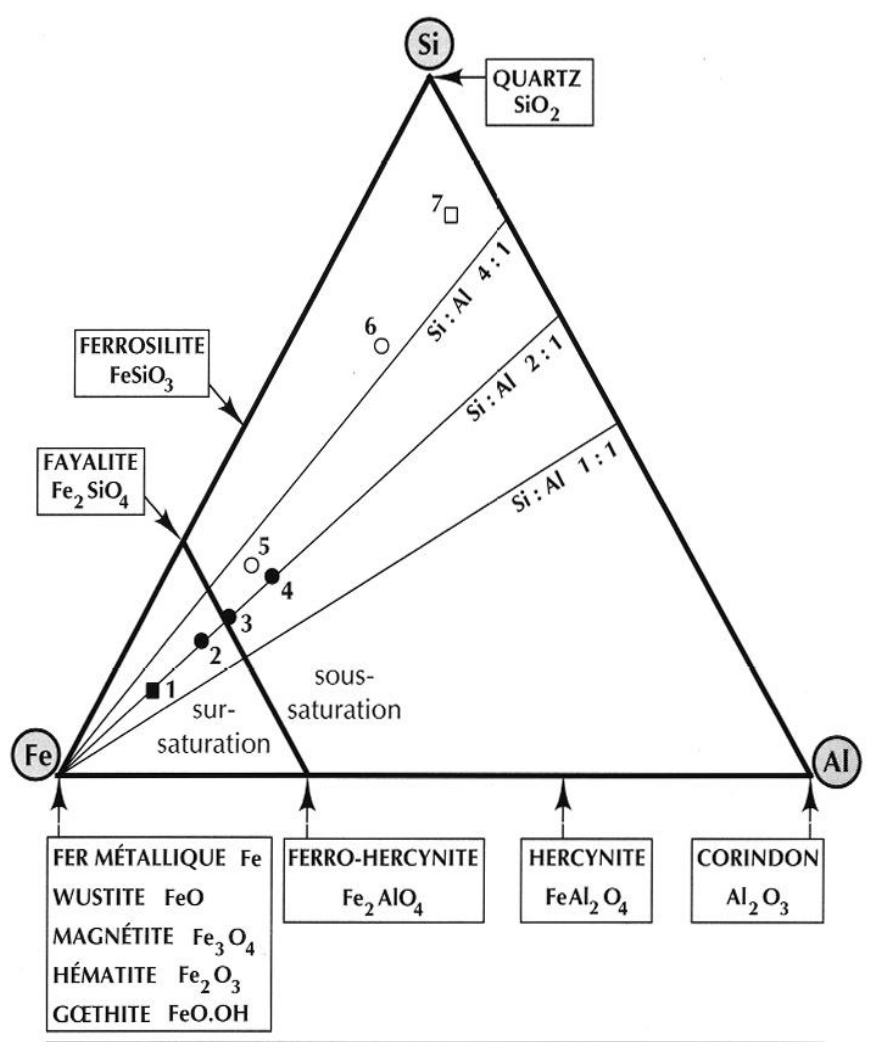

\begin{tabular}{|ll|}
\hline \multicolumn{2}{|c|}{ PROJECTION EN POURCENTAGES DE CATIONS } \\
\hline 1 minerai & \\
2 scorie sursaturée en oxydes de fer & 5 scorie contaminée en silice \\
3 scorie saturée en oxydes de fer & 6 paroi contaminée en oxydes de fer \\
4 scorie sous-saturée en oxydes de fer & 7 paroi siliceuse \\
\hline
\end{tabular}

Fig. 44 - Traitemenl graphique à l'aide d'un diagramme triangulaire Si-Al-Fe (d'après Serneels, 1993, p. 29-30). Les valeurs analytiques sont converties en cations. Les analyses d'un minerai et des scories qui sont issues de la réduction de ce minerai se projettent normalement sur une droite passant par le pôle Fe. Sil y a une contamination due par exemple à la fusion de la paroi du fourneau, le rapport Si: Al est modifié et les scories sont décalées par rapport au minerai. La distance qui sépare la projection du minerai et celle de la scorie est proportionnelle à la quantité de fer produite. On définit une limite de saturation en oxydes de fer matérialisée par la droile fayalite/ferrohercynite. Les scories qui se projettent vers le pôle Fe sont considérées comme sursaturées, c'est-à-dire qu'elles contiennent potentiellement des oxydes de fer libres, non combinés avec Si ou Al (Serneels, 1993). La réduction est assez mauvaise. Les scories qui se projettent au-delà de la limite de saturation témoignent d'une réduction plus efficace. Cette représentation ne tient pas compte de la présence d'autres éléments comme le phosphore, le calcium ou le manganèse. Elle est inadaptée lorsque ces éléments sont abondants.

associations minéralogiques et leurs conditions de formations. Malheureusement ils ne sont pas connus pour tous les cas et ceux qui ont été établis ne concernent que des compositions simples, à un, deux, trois, parfois

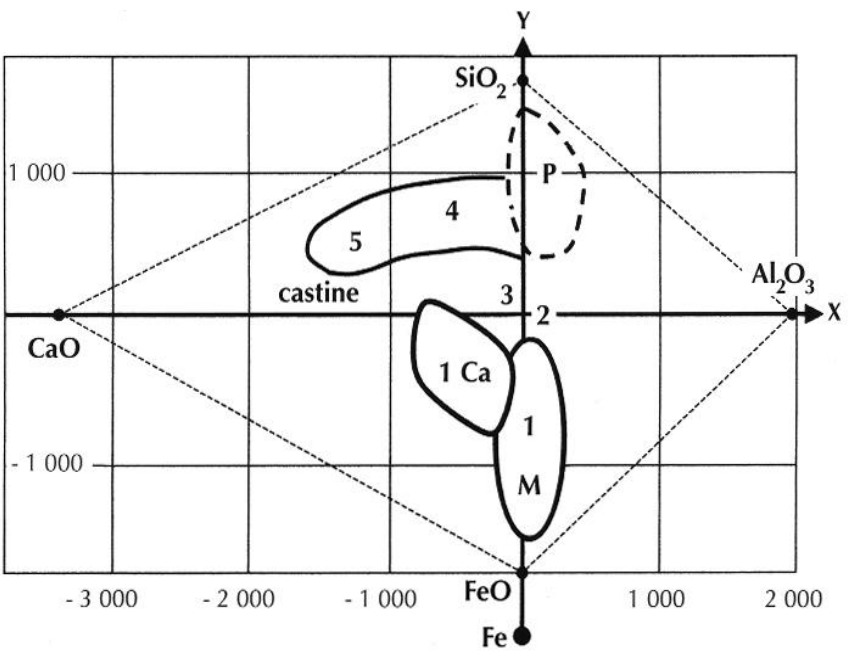

Fig. 45 - Diagramme paramétrique chimico-minéralogique (La Roche, 1972) mis au point pour une représentation des compositions des minerais de fer, des scories et produits associés en sidérurgie (d'après Ploquin, 1992, 1993, 1994a). Les paramètres X et $Y$ sont tels que ce diagramme est équivalent à une projection du tétraèdre silice-ferchaux-alumine, représenlatif des principaux éléments constituant scories et minerais de fer, corrigé des alcalins de manière à regrouper les matériaux des parois sur l'axe des $Y(>>0)$ ou à sa droite. $X=A l-(N a+K+2 \mathrm{Ca}) ; Y=S i-F e$ exprimés en milli-atomes dans $100 \mathrm{~g}$. Conversion des pourcentages masse d'oxyde en milli-atomes dans $100 \mathrm{~g}: \mathrm{Si}=\mathrm{SiO}_{2} \times 16,65 ; \mathrm{Al}=\mathrm{Al}_{2} \mathrm{O}_{3} \times 19,619 ; \mathrm{Fe}=\mathrm{Fe}_{2} \mathrm{O}_{3}$ $\times 12,523+\mathrm{Fe}_{2} \mathrm{O} \times 13,918 ; \mathrm{Ca}=\mathrm{CaO} \times 17,832 ; \mathrm{Na}=\mathrm{Na}_{2} \mathrm{O}$ $x 32,258 ; \mathrm{K}=K_{2} \mathrm{O} \times 21,231$; $P$, revêtements internes de parois; $M$, minerais (les minerais calciques sont en $1 C a$, partie gauche); 1, scories denses fayalitiques (les faciès riches en wustite et/ou en fer métallique sont en bas); $1 \mathrm{Ca}$, scories denses calciques; 2 , scories mi-lourdes; 3 , quasi-laitiers (nous avons longtemps nommé ces deux types "scories légères"); 4, laitiers siliceux et silico-calciques des hauts fourneaux archaïques et anciens (charbon de bois); 5, laitiers hypercalciques des hauls fourneaux récents.

quatre éléments, rarement plus ${ }^{115}$. Cependant ils sont précieux, par exemple pour discuter l'ordre de cristallisation de minéraux à partir d'un bain fondu: minéralogie des scories, confrontation avec les observations au microscope. Ainsi, le triangle $\mathrm{CaO}-\mathrm{SiO}_{2}-\mathrm{Al}_{2} \mathrm{O}_{3}$ représente la gangue d'un minerai ou peut s'appliquer à un laitier de haut fourneau. Les triangles $\mathrm{CaO}-\mathrm{SiO}_{2}-\mathrm{FeO}$ ou $\mathrm{Al}_{2} \mathrm{O}_{3}$ $\mathrm{SiO}_{2}-\mathrm{FeO}$ seront applicables à des produits calciques ou alumineux mais non calco-alumineux (fig. 44).

115. M. Levin et al. (ed.), Phase diagrams for ceramists, The American Ceramic Society, Colombus, 1956, 1959, 1964, 1969 et Verein Deutscher Eisenhüttenleute, Schlackenallas/Slagatlas, Düsseldorf, Verlag Stahleisen MBH, 1981, $282 \mathrm{p}$. 
La combinaison de ces quatre règles permet de suivre dans des diagrammes chimiques adaptés l'évolution des compositions ou, inversement, de tenter de remonter aux compositions initiales à partir des produits trouvés. Divers diagrammes sont adaptés à la typologie chimique en première approximation (fig. 45) (Ploquin, 1993; Mahé-Le Carlier, Ploquin, à paraître) et à la distinction locale de travaux artisanaux (Chardron-Picault, Pernot, 1999, p. 283-285).

\section{Principes de bases relatifs aux textures}

Nous appelons textures les figures que forment les minéraux, ou toute autre phase, et leurs relations mutuelles. Aucun manuel dédié à la pétrographie des scories n'est disponible; quelques publications donnent des exemples, pas toujours bien argumentés. En revanche, il existe des ouvrages consacrés aux minerais géologiques.

La première observation au microscope pétrographique consiste à examiner si la lame ou section est homogène (i.e. même association et même morphologie sur toute la surface); il faut définir chaque zone homogène: nature et morphologie (formes et tailles) des phases, relations mutuelles, proportions; puis déterminer s'il s'agit d'une association ou de phases formées à partir d'un matériau solide, fondu, ou mixte. Dans le cas d'un matériau resté solide, quels sont les produits initiaux et les produits de réaction, y a-t-il changement de volume ou de forme? Dans le cas d'un matériau fondu, les phases présentes ont-elles cristallisé à partir de ce bain (cristaux à forte tendance automorphe), ou se sont-elles formées par solidification d'une phase liquide séparée (démixtion) du bain (exemple billes de fonte). Certes, il existe des cas ambigus susceptibles d'alimenter des discussions. La morphologie des cristaux peut être un indicateur des modalités de cristallisation: en première approximation on assimile les morphologies dendritiques, formées dans un bain refroidi nettement en dessous de son liquidus, à une cristallisation rapide, plus rapide que pour les formes squelettiques et les formes plus trapues mais idiomorphes refroidies près du liquidus. Les formes trapues semblent bien caractéristiques des scories refroidies dans le four.

Il faut aussi, le cas échéant, définir les rapports entre chaque zone (nature et morphologie des contacts) et tenter de déterminer s'il s'agit d'inclusions, d'états reliques d'un matériau incomplètement « assimilé » (ex. reliques de croûtes ou de gouttes de métal oxydé dans une scorie de forge ou d'épuration ou d'affinage, reliques de minerai, etc.), de non miscibilité de deux liquides, d'immiscibilité temporaire au sein d'un liquide, de ségrégations gravitaires ou thermiques, de limites entre écoulements, etc.

\section{Conclusions et remarques}

De tout ce qui précède, et c'est loin d'être exhaustif, le lecteur comprendra que la sélection des spécimens est très importante et qu'elle doit être conduite en collaboration entre le fouilleur et l'archéomètre pétrologiste (pétrologie $=$ pétrographie et géochimie), afin de replacer au mieux les observations dans leur contexte, l'appareil (four, foyer, etc.) ou l'atelier et de relativiser leur importance. Remarquons que les spécimens pétrographiquement les plus significatifs ne sont pas forcément les plus représentatifs; une "relique " pétrographique, souvent peu fréquente, sera riche d'informations sur la genèse du spécimen. En revanche, la notion de représentativité est essentielle pour l'établissement de bilan matière.

En résumé nous proposons la typologie chimicominéralogique suivante (Ploquin et al., 1996; MahéLe Carlier, 1997):

Type 1. Scories "denses " à fayalite (ou kirschteinite si elles sont calciques, ou mélilite si elles sont calcoalumineuses) ( \pm spinelle de la série hercynite-magnétite si elles sont alumineuses) et wustite, fer amiboïde ou dendroïde.

Type 2. Scories " mi-lourdes" à fayalite ( \pm spinelle), wustite localisée ou quasi absente, fer en billes ou petits cristaux; souvent manganésifères.

Type 3. Scories « légères" ou " quasi-laitiers » à mélilite, pyroxénoïde, voire pyroxène, parfois olivine, fer en billes (teneur en $\mathrm{C}$ variable); teneur en $\mathrm{Fe}_{2} \mathrm{O}_{3}$ total supérieure à 20-30\%. Ces produits sont les scories classiques des bas fourneaux, généralement largement cristallisés sauf le type 3 qui présente souvent une phase vitreuse plus conséquente.

Type 4. "Laitiers " à pyroxénoïde ou pyroxène, fer en billes (fonte ou variable); teneur en $\mathrm{Fe}_{2} \mathrm{O}_{3}$ total de $15-20 \%$ à très peu, teneur en $\mathrm{CaO}$ inférieure ou du même ordre que $\mathrm{SiO}_{2}$. Ce type peut apparaître dans quelques (grands?) bas fourneaux (exemple Oulches, 
Berry, cf. Mahé-Le Carlier et al., 1998), mais il est plus typique des hauts fourneaux archaïques $\left(\mathrm{Fe}_{2} \mathrm{O}_{3}\right.$ total soutenu) ou plus récents, mais fonctionnant au charbon de bois $\left(\mathrm{Fe}_{2} \mathrm{O}_{3}\right.$ total moindre), souvent avec un usage modéré, voire variable, de castine (i.e. ajout de calcaire).

Type 5. « Laitiers hypercalciques » à minéraux de type larnite, merwinite, rankinite, fer en billes (fonte) ; teneur en $\mathrm{CaO}$ supérieure à $\mathrm{SiO}_{2}$. Ce type correspond aux hauts fourneaux récents, plus grands, fonctionnant au charbon (coke), avec un fort ajout de castine. Alors que le type 4 est souvent vitreux ou partiellement vitreux, le type 5 est le plus souvent cristallisé (dans des traités du XIX ${ }^{c} s$. on trouve les expressions de « laitiers filants » et de « laitiers courts $»$ ).

Remarquons que les scories de forge (faciès $b$, in: Dunikowski et al., 1996) et les scories d'affinage (de la fonte) ressemblent beaucoup au type 1, voire 2. En effet elles sont produites par réactions entre le métal, plus ou moins oxydé, et divers produits: ajouts, cendres, impuretés du métal. Des parois fondues ou des ajouts de forge fondus peuvent ressembler à des laitiers 3 (réactions avec scories) ou 4. Des différences chimiques, souvent minimes, et des faits pétrographiques aideront à faire le diagnostic. La contamination des scories par les parois est rarement importante alors que l'inverse est plus net.

\section{APPROCHE DE TYPE MÉTALLOGRAPHIQUE}

Le métal est la cible privilégiée: sa nature, ses états, ses textures et morphologies, ses relations avec les formes oxydées. L'expérience du métallographe sera fondamentale.

\section{INDICES DE RÉDUCTION DIRECTE}

Le métal (fer-acier) est intimement mêlé à la matrice silicatée de la scorie fayalitique et peut prendre différents aspects.

- Il peut se présenter en globules souvent monocristallins, distribués en filaments tortueux, voire anastomosés, et/ou en chapelets localisés à proximité de vecteurs réducteurs (charbon de bois, puits de diffusion, bulle gazeuse, $c f$. Fluzin, 1994). Cette disposition peut même correspondre aux anciens grains de minerai comme l'illustre le cas le plus visuel du minerai oolithique lor- rain (Leroy, 1997; Leroy, Merluzzo, 2000) (fig. 46, n 1). L'agglomération de ces globules métalliques est plus ou moins importante en fonction de l'élimination de la scorie interstitielle: processus " d'assèchement de l'éponge de fer » (fig. 46, $\mathrm{n}^{\circ} 2$ ).

- Il peut prendre l'aspect d'un polycristal avec agglomération centripète de petits globules à proximité ou non d'une porosité (Fluzin et al., 1995).

- Il peut également se développer au sein de la wustite (réduction partielle ou totale), y compris lorsque celle-ci présente un aspect dendritique (Fluzin, Leclère, 1998).

- La morphologie des grains (éléments métalliques) est le plus souvent peu anguleuse et ne présente pas d'écrouissage particulier dans le cas des polycristaux.

- Il est possible d'observer (cas assez rare au stade de la réduction) la présence de quelques globules apparaissant comme sphériques au niveau du plan de coupe. Cette morphologie est d'autant plus vraisemblable lorsque la teneur en carbone augmente, compte tenu de l'abaissement du point de fusion de l'alliage fer-carbone. Elle est à associer à des niveaux de température élevés (proximité de la tuyère, gros bas fourneaux). La décarburation de l'acier pourrait également justifier ce faciès. Sans exclure l'observation en réduction de telles configurations, elles sont toutefois plus fréquentes dans le cas des opérations de forge.

- Le métal peut présenter des porosités (de morphologie bien souvent caractéristique), plus ou moins vides en fonction du degré d'élimination des scories. Associés à certaines de ces porosités (notamment les porosités vides), il n'est pas rare d'observer des replis de métal (fig. $46, n^{\circ} 3$ ). Ces derniers résultent des phénomènes de coalescence et d'agglomération progressifs du métal qui se densifie au fur et à mesure de l'évacuation de la matrice silicatée (fig. $46, \mathrm{n}^{\circ}$ 2). La scorie liquide s'écoulant, cela favorise la jonction des différentes parcelles métalliques, ce qui engendre, suivant le contexte, la formation de replis qui sont relativement gros et mal formés en début de processus et disparaissent progressivement au fur et à mesure du traitement thermomécanique d'épuration. Ces replis sont donc fréquemment observés dans le métal des loupes, mais aussi dans les divers déchets inhérents aux différentes phases de l'épuration (gromps, culots...). Notons que certains d'entre eux subsistent et semblent aussi apparaître dans quelques cas de forge d'élaboration d'objets. 


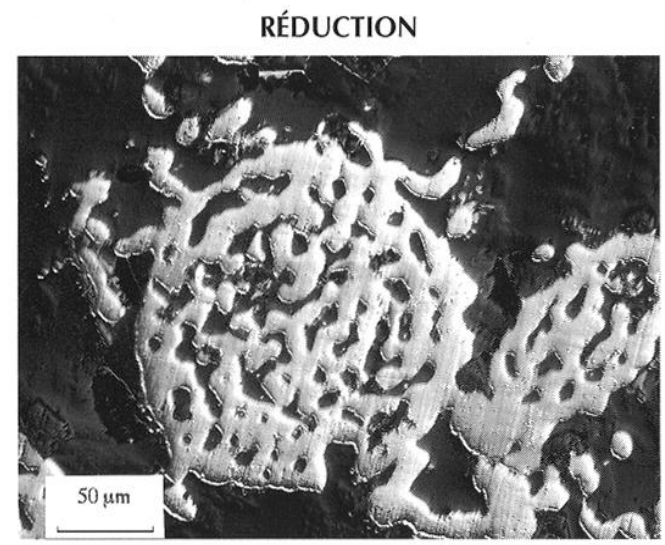

1

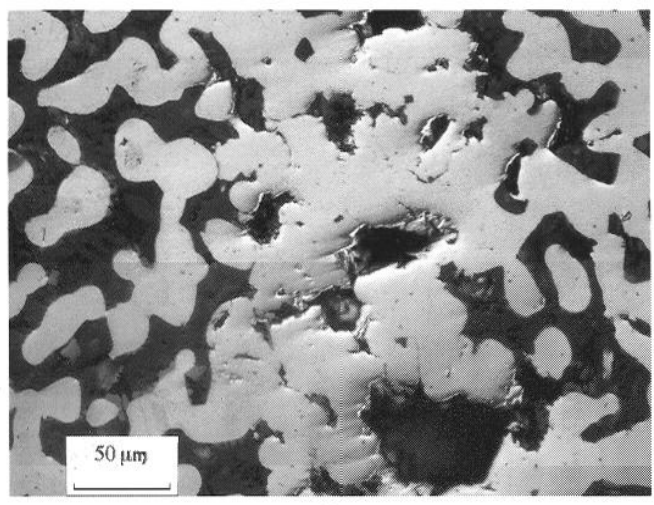

2

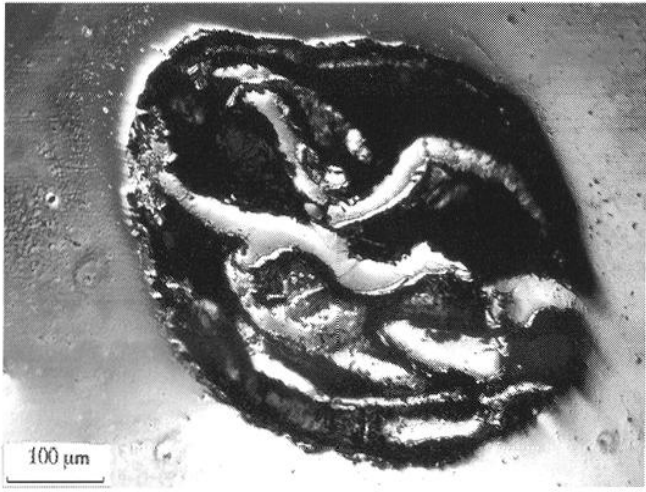

3

Fig. 46 - Processus de transformation du métal, indices mélallographiques: 1, minerai oolithique lorrain, au cours de la réduction le métal apparaît en gardant la forme de l'oolithe (expérimentation de réduction el d'épuration: Nancy-Beljort, 1995, P. Fluzin, I. Leclère, M. Ieroy, P. Merluzzo; cliché P. Fluzin); 2, agglomération et densification du métal dans une loupe, maléniel ethnoarchéologique (expérimentation: site de Ardingi, Mali, 1995, E. Huysecom, V. Serneels, I. Fluzin; cliché P. Fluzin); 3 , replis mélalliques au cours de l'épuration d'une loupe (expérimentation: Belfort, 1995, D. Leclère, P. Fluzin; cliché P. Iluzin).

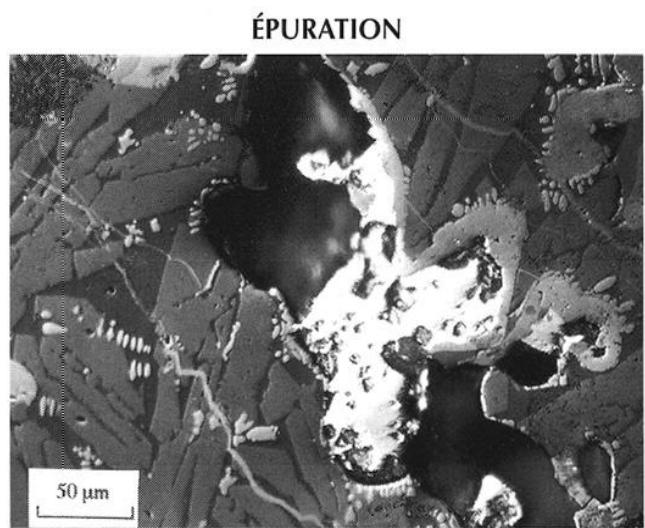

1

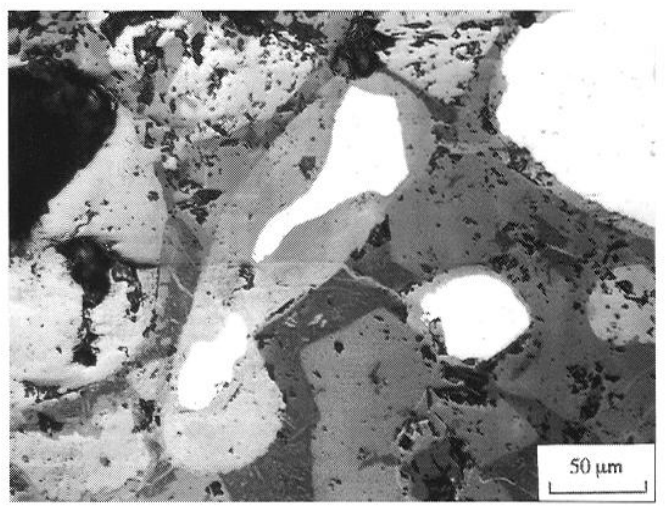

2

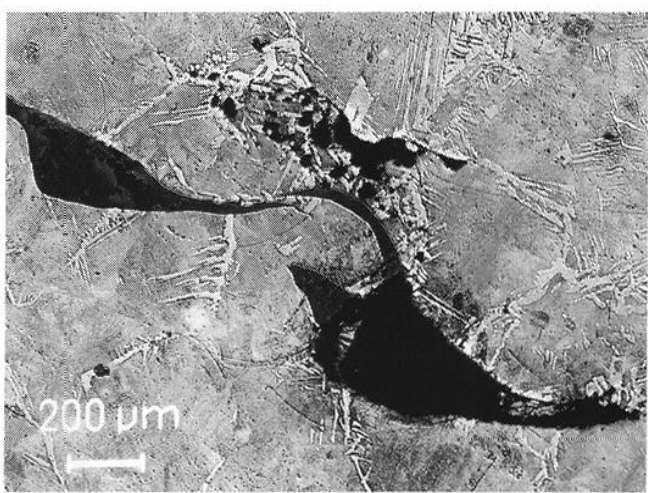

3

Fig. 47 - Pmcessus de transformation du métal, indices métallographiques: 1, fragment de fer déchiqueté en inclusion dans une porosilé, culol archéologique gallo-romain (site de Touffreville, Calvados, 1995, N. Coulthard, P. Fluzin; cliché $P$. Fluzin); 2, scories avec éléments mélalliques en cours de réoxydation à chaud (échantillon 07b, poids $160 \mathrm{~g}$ : site de

Vic-de-Chassenay, Morvan, d'après Mangin et al., 2000a; cliché $P$. Fluzin); 3, repli en cours d'écrasement avec remplissage. partiel de silice, lingot archéologique (centre) (sile de Coulmier-le-Sec, Côte-d'Or, d'après Mangin et al., 2000a; cliché P. Fluzin). 


\section{INDICES DE FORGE D'ÉPURATION : TRAVAIL DU MÉTAL BRUT}

Nous avons déjà signalé (supra, p. 108) la présence des gromps et des battitures au voisinage de zones de martelage (cinglage).

Dans les scories, généralement en grosses calottes, ces indices sont sans aucun doute les plus difficiles à mettre en évidence, compte tenu de la persistance éventuelle d'indices de réduction, d'une part, et de leur convergence avec les indices de forge d'élaboration d'objets, d'autre part. Les opérations à la forge sont caractérisées par des alternances de réchauffage au sein d'un foyer où l'atmosphère sera très riche en $\mathrm{CO}$ et de martelage à chaud et à l'air (cycles thermomécaniques).

L.e métal «perdu " au cours de l'opération d'épuration n'est pas négligeable, comme l'indiquent les reconstitutions expérimentales. I.e nombre et la taille des fragments sont plus importants en début d'opération. Ceci doit par exemple se traduire dans la répartition du métal au sein d'une calotte, si elle n'a pas été trop homogénéisée. Compte tenu des spécificités thermomécaniques de cette opération, les fragments métalliques se retrouvant au sein des calottes peuvent présenter différents aspects conjugués ou dissociés:

- une répartition aléatoire de fragments aux contours relativement déchiquetés (angles vifs) dont la liaison avec la matrice n'est pas continue (fig. $47, \mathrm{n}^{\circ} 1$ ) ;

- une masse métallique plus importante et plus compacte correspondant à la perte de gros morceaux (début d'opération);

- une réoxydation interne du métal (à proximité de puits de diffusion) ou périphérique, caractéristique de la forge (fig. $47, \mathrm{n}^{\circ} 2$ ) ; cette réoxydation peut s'accompagner dans le cas d'un acier d'une décarburation superficielle; pour mémoire, l'opération inverse de rémentation nécessite des durées de traitement très longues (10 heures, $c f$. Fluzin, 1994);

- la présence de petits replis métalliques déformés, imparfaitement soudés au sein d'un morceau de métal, dénotant un forgeage insuffisant (fig. $47, \mathrm{n}^{\circ} 3$ ) ;

- la présence locale de métal écroui au contour irrégulier;

- la présence éventuelle de battitures (ou plus correctement dit de vestiges de croûtes d'oxydation) dans les culots, bien que celles-ci se situent généralement à proximité de l'aire de martelage;

- la présence de billes ou de chapelets de métal (feracier) partiellement ou non réoxydés. La non réoxyda- tion de ces éléments métalliques peut s'expliquer par des conditions locales limitant les réactions: le métal se situe dans un environnement relativement protectcur (tampon) de scories. Les reconstitutions expérimentales que nous avons effectuées ont montré que des billes se situaient préférentiellement à proximité d'une source thermique élevée (tuyères).

\section{INDICES DE FORGE D'ÉLABORATION D'OBJETS: TRAVAUL DU MÉTAL ÉPURÉ}

Comme pour le cas de la forge d'épuration, les battitures se situent principalement à proximité de l'aire de martelage (fig. $48, \mathrm{n}^{\circ} \mathrm{l}$ ). Elles sont généralement prédominantes par rapport aux autres déchets. Leurs dimensions sont en fonction de la surface du métal travaillé: l'élaboration d'un objet de petite dimension engendre de petites battitures. Ce qui n'est pas le cas pour le travail de surfaces plus importantes, comme par exemple l'élaboration d'une épée comme en témoigne la reconstitution que nous avons effectuée pour les épées de Gournay-sur-Aronde (Fluzin et al., 1983). Des observations faites dans des taillanderies encore en activité ou en démonstration (Nans-sous-Sainte-Anne, Pont-Salomon, Marthod, Bienno, etc.) tendent à montrer que leur épaisseur est fonction de la violence du choc. Dans le cas de l'utilisation d'ajouts, elles peuvent avoir une surface à cratères (Dunikowski et al., 1996). Mais, différence prévisible avec les opérations d'épuration, elles seront plus abondantes et les gromps seront absents.

L'environnement de la forge, en dehors des culots et des battitures, peut également livrer un matériel plus ou moins abondant constitué de fragments de métal généralement oxydés. Il peut s'agir de chutes, d'ébauches d'objets, de soies de préhension, de fragments d'outils, ou de morceaux de métal éventuellement destinés à être réutilisés. L'étude morphologique et métallographique de ce matériel peut contribuer à préciser le type d'activité (Dunikowski et al., 1996). Il est en outre possible d'évaluer le degré de compétence technique des artisans (traitements thermiques, etc.). À cet égard, il convient de faire remarquer que la réalisation de simples tôles de métal (armure, fourreau d'épée, etc.) nécessite de la part $\mathrm{du}$ forgeron une maitrise technique plus grande (ainsi qu'un métal de meilleure qualité) que l'élaboration d'objets massifs. En effet, l'amincissement d'une tôle est très difficile à exécuter en forge artisanale. 
MISE EN FORME

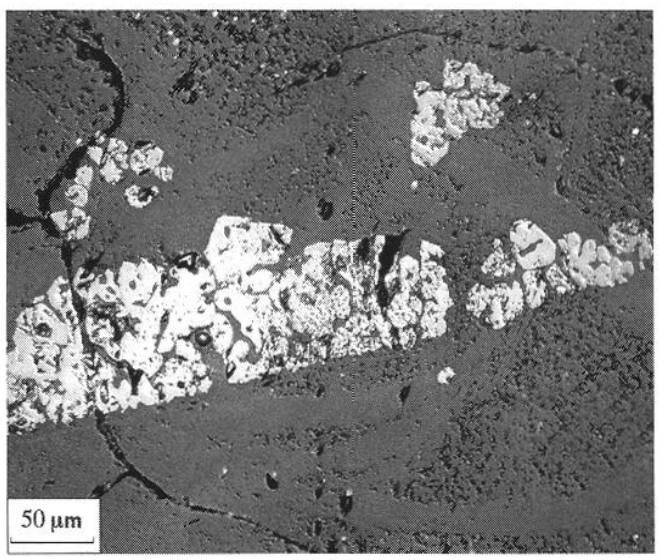

1
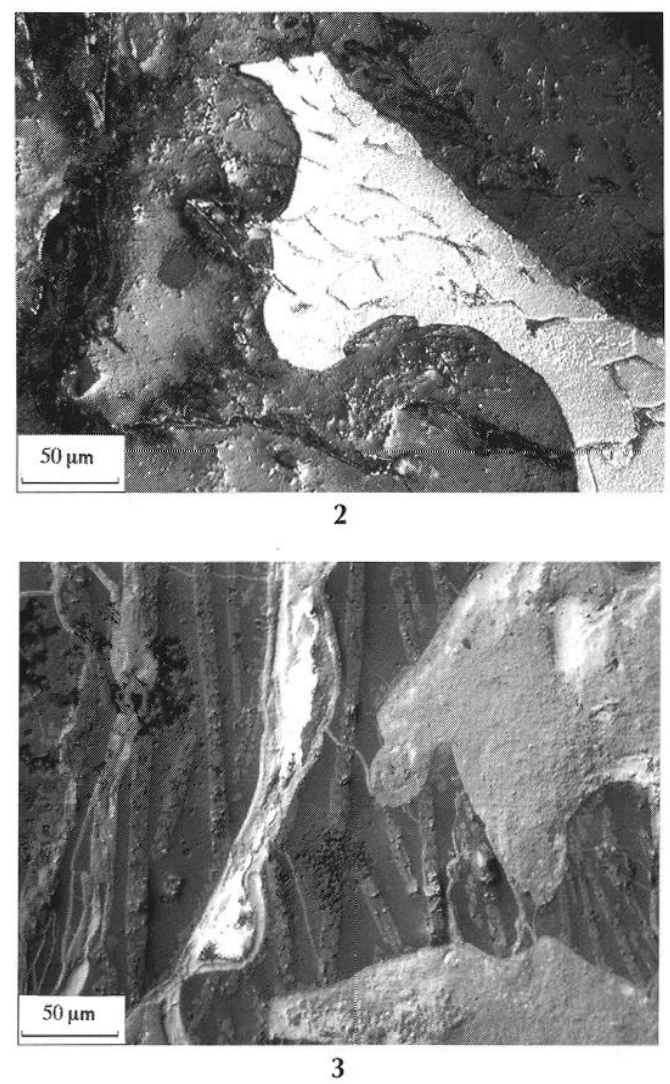

Fig. 48 - Processus de transformation du mélal, indices mélallographiques: 1, relique de croûte et de gouttes oxydées ou de baltiture dans un culot archéologique de forge (second âge du Fer) (site de Puy-de-Crâce, Corrèze, 1997, C. Besl, P. Fluzin; cliché P. Fluzin); 2, fragment de fer écroui dans un culot archéologique gallo-romain (site de Touffreville, Calvados, 1995, N. Coulhard, P. Fluzin; cliché P. Fluzin);

3, filament de fer complètement réoxydé, scorie archéologique (site de Juude-Jaabe, Sénégal, 1995, H. Bocoum, P. Fluzin; cliché P. Fluzin).
Dans le cas d'une opération découplée de la forge d'épuration, nos expérimentations et des observations dans des forges « à l'ancienne » nous conduisent à formuler les remarques suivantes:

- lorsqu'il cherche à élaborer des objets de bonne qualité, le forgeron a intérêt à travailler avec un foyer le plus propre possible pour bien contrôler son travail (estimation visuelle de la température, éviter les pollutions) et à concentrer le feu en focalisant le foyer suivant le type d'objet à travailler; ceci nécessite un nettoyage régulier du foyer; les déchets qui sont déjà relativement faibles (forge au charbon de bois) sont, semble-t-il, par conséquent de taille plus modeste qu'en épuration;

- la fréquence des cycles thermiques est plus élevée (en fonction du type de fabrication) et ceux-ci sont de durée relativement faible: il faut éviter que le métal ne brûle dans le cas d'un acier et limiter les pertes au feu; ceci se traduit par une plus grande homogénéité des culots (atténuation de la stratification);

- les pertes au feu pour produire un objet (pointerolle, épée) sont de l'ordre de quelques pour cents du poids initial de métal (lingot) par cycle; elles sont nettement plus faibles qu'en forge d'épuration; ce métal, lorsqu'il est retrouvé, présente souvent, en fonction de ses conditions de chauffage (niveau de température atteint) et de refroidissement, un écrouissage important et est en liaison intime avec la matrice (fig. $48, \mathrm{n}^{\circ} 2$ ); il faut toutefois considérer l'exception de la forge "de recyclage ", où l'on procède à de nombreuses soudures d'éléments de tailles variables pouvant engendrer des pertes de métal non négligeables dans le foyer de forge; il n'est pas rare d'observer une décarburation en périphérie des éléments métalliques les plus riches en carbone;

- le métal brûlé génère un faciès métallographique assez. caractéristique; il est possible de le retrouver en chute mais aussi en inclusion reprise dans une scorie (calotte); - comme c'est indiqué dans le cas de l'épuration, des croûtes oxydées peuvent tomber dans le foyer et être incorporées à la scorie ; au «blanc suant ", des gouttes de métal, plus ou moins oxydé, pourront être incorporées (fig. $48, \mathrm{n}^{\circ} 1$ ).

Les soudures présentent un certain nombre de spécificités selon la teneur en carbone du métal ${ }^{116}$. En effet,

116. I. Guillot, P. Fluzin, Interprétation structurale de l'élaboration et de l'utilisation d'outils miniers, Bulletin de la Société préhistorique franşaise, 84, crsm 8, 1987, p. 248-256; I. Guillot, P. Fluzin, M. Clavel, G. Béranger, Structure d'outils miniers du XVe et du XVIII' siècle. Interprétation 
pour réaliser une soudure la température requise est celle du " blanc soudant ", vers $1400-1450^{\circ} \mathrm{C}$, pour le fer. La tranche de température pendant laquelle le métal est susceptible de se souder dans les conditions ordinaires est appelée palier de soudabilité. Il est plus long et plus haut pour le fer que pour l'acier (la température de fusion du métal décroît quand sa teneur en carbone croît). La soudure acier sur fer est donc plus difficile à réaliser car il faut que les deux métaux soient amenés en même temps à leur palier de soudabilité, ce qui exige un écart de température. La formation d'oxyde sur les surfaces à souder empêche le contact intermétallique; pour l'éliminer, il est nécessaire, à chaud, de parsemer de décapant (sable ou poudre de minerai, cendres, etc., maintenant borax) les surfaces à réunir, de façon à limiter l'oxydation et à faciliter l'éclatement de la couche d'oxyde. Le sable forme alors avec l'oxyde de fer un silicate fluide à haute température qui est évacué par martelage sous forme de battitures et de scories et dont les restes constitueront les alignements d'inclusions au niveau des soudures (Fluzin et al., 1983; Dunikowski et al., 1996).

La réalisation de traitements thermochimiques (cémentation, nitruration, trempe sélective...) nécessite l'emploi de substances approprićcs (matières organiques, argiles...) qui pourront se retrouver en partie dans les culots.

Nous ne détaillerons pas davantage les résultats obtenus à l'aide de cette méthode. Bien que se limitant à la "lecture du métal " dans son environnement (objet, culot, scorie), elle permet de travailler à moindre coût sur de gros échantillons et de déterminer des indices propres à identifier l'activité sidérurgique ainsi que de découvrir le mode de fabrication d'un objet.

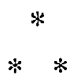

Cet article n'est pas un manuel d'archéométrie mais une présentation du champ des investigations archéo- métriques permettant d'essayer de restituer les différentes étapes de la chaîne opératoire en sidérurgie à partir des déchets retrouvés par les archéologues.

Les études métallographiques ne se cantonnent plus maintenant à l'étude des objets, manufacture et réparations, mais s'appliquent aussi à la genèse et à la mise en ceuvre du métal, et à la reconnaissance des étapes. La notion d'héritage chimique et pétrographique permet de reconstituer, au travers d'un déchet, les apports des matériaux parents (scories de réduction/ minerai avec ou sans ajouts; scories de forge/ajouts et métal; etc.) et leurs transformations. Les processus physico-chimiques mis en ouvre sont étudiés et leur compréhension permet d'introduire plus de cohérence dans nos reconstitutions.

Nous avons montré la pertinence des résultats obtenus depuis plusieurs années grâce à une collaboration interdisciplinaire réelle: sciences de l'homme et de la société, sciences naturelles, sciences des matériaux. Chaque méthode, chaque discipline a ses limites et seule leur confrontation permet de " faire parler » les vestiges archéologiques en minimisant les sources d'erreur. La compréhension des vestiges sidérurgiques contribue à l'histoire des sciences et techniques, mais ouvre aussi des perspectives sur les dimensions économiques et sociales de cette industrie, donc sur l'histoire en général. Par ailleurs, les scories et les métaux anciens fournissent aux ingénieurs des analogues réels qui permettent de comprendre les phénomènes de vieillissement des matériaux, connaissance applicable dans les domaines du génie civil, de la conservation du patrimoine ou encore de la gestion des déchets modernes. Des anomalies structurales observées dans les métaux anciens laissent entrevoir de nouveaux matériaux, comme ceux qui sont élaborés par mécanosynthèse. Malgré tous nos efforts et nos succès, il convient de rester modeste tant "l'objet technique, traduction physique d'un système intellectuel, n'est jamais complètement connu» (Simondon, 1969) ${ }^{117}$.

Interprétation thermomécanique, Matériaux el Techniques, 10-11, 1987, p. 411-419; I. Guillot, P. Fluzin, P. Benoit, P. Béranger, Études paléométallurgiques comparatives d'outils miniers du XV' et du XVII siècle, in: Archäometallurgie von Kupfer und Eisen in Westeuropa, Actes du symposium de Mayence, 12-15 sept. 1986, Jahrbuch des Römisch-Germanischen Zentralmuseum Mainz, 35, 1988, p. 641-655.

117. Tous les collègues, professionnels ou bénévoles, archćologues, historiens, métallurgistes ou géologues, avec lesquels nous avons des collaborations et échanges, notamment au sein de l'Association archéologique pour l'étude des mines et de la métallurgie et de séminaires, ont de fait apporté leurs contributions au bilan présenté. Claude Domergue et Marc Lcroy nous ont bien aidés à élaguer et recentrer nos propos. 